\title{
Aporte del SENA al Sistema Nacional de Ciencia, Tecnología e Innovación de Colombia
}

\author{
The Contribution of SENA to Colombian Science, \\ Technology, and Innovation System
}

\section{Gustavo Vargas Yara ac, Darwin Dubay Rodríguez ${ }^{\text {ad }}$, Aylin Viviana Silva Ortiz ${ }^{\text {be }}$}

\author{
${ }^{a}$ Grupo de Investigación del Centro Metalmecánico (GICEMET), Servicio Nacional de Aprendizaje (SENA), Colombia \\ ${ }^{b}$ Asesora Servicio Nacional de Aprendizaje (SENA), Colombia \\ c gvargas@sena.edu.co | https://orcid.org/0000-0003-4733-2136 \\ d drodriguezp@sena.edu.co| https://orcid.org/0000-0003-1172-1295 \\ e avsilva@sena.edu.co| https://orcid.org/0000-0001-6610-5188
}

\section{RESUMEN}

Existen diferentes enfoques que dan cuenta de la importancia de la relación entre el crecimiento económico y la implementación de programas de ciencia, tecnología e innovación (CTI) en el marco del diseño, la ejecución y la evaluación de políticas públicas. Por lo anterior, este trabajo aborda dicha relación desde el concepto, la metodología, la normativa y la implementación de la política de CTI de Colombia, resaltando el aporte del Servicio Nacional de Aprendizaje (SENA) a la construcción de programas de CTI en el país. Aquí se muestran las contribuciones de la estrategia SENNOVA, creada por el SENA, como respuesta institucional a la normatividad de ciencia y tecnología nacional y la organización en la inversión de recursos. Finalmente, se concluye que SENNOVA es un agente multiplicador de la apropiación tecnológica, social y colectiva, al transferir el conocimiento generado en los proyectos de diferentes líneas programáticas de CTI a los procesos de formación profesional y al sector pro-

Citation: Varas-Yara, G., Rodríguez, D. D. y Silva-Ortiz, A. V. (2021). Aporte del SENA al sistema nacional de ciencia, tecnología e innovación de Colombia. Mutis, 11(2), 109-131.. https://doi.org/10.21789/22561498.1723

Recibido: 23 de febrero de 2021. Aceptado: 30 de abril de 2021.

Copyright: $\odot 2021$. Varas-Yara, G., Rodríguez, D. D. y Silva-Ortiz, A. V. (2021). This is an open-access article, which permits unrestricted use, distributions and reproduction in any medium, provided the original author and source are credited.

Competing Interests: The authors have no conflict of interest. ductivo, contribuyendo así con la disminución de las brechas tecnológicas existentes en el país.

Palabras clave: SENNOVA, SENA, ciencia, tecnología, innovación.

\section{ABSTRACT}

Different approaches remark the importance of the relationship between economic growth and the implementation of science, technology, and innovation programs within the design and evaluation of public 
policies. Therefore, this paper addresses said relationship from the concept, methodology, regulations, and implementation process of Colombian policy regarding science, technology, and innovation, highlighting the contribution made by the National Apprenticeship Service (SENA) to the development of related programs. The contributions made by the SENNOVA strategy, created by SENA, as an institutional response to national regulations on science and technology and the allocation of resources are also reported in this work. We concluded that SENNOVA is a multiplying agent of technological, social, and collective appropriation given its role in transferring the knowledge generated in the projects from different programmatic lines of science, technology, and innovation to professional training and the productive sector, thus contributing to the reduction of technological gaps in the country.

Keywords: SENNOVA, SENA, science, technology, innovation.

\section{INTRODUCCIÓN}

La creación de políticas públicas para fomentar la ciencia, la tecnología y la innovación (CTI) como actores importantes del desarrollo productivo de una nación se encuentra determinada por agentes externos que muchas veces están influenciados por el tipo de régimen político. Este es el caso de Ghana, economía emergente de África, donde desde 1957 hasta 2012 se identifican cambios en la política de CTI y los efectos de esta según el régimen político de turno, como muestra Amankwah-Amoah (2016). En países más desarrollados se siguen estructuras de política pública basadas en instrumentos que permiten su rápida y eficaz implementación, así como la pronta modificación de dicha política en caso de que esta no funcione. Generalmente, en estas naciones se guarda una total coherencia entre los objetivos de desarrollo establecidos en la política general del país y la planificación, distribución de recursos, prospectiva de ciencia y tecnología, y los instrumentos para tal fin, haciendo un riguroso seguimiento y control a los resultados, con un menor efecto generado por el régimen de turno (Peijie \& Li, 2021). En países vecinos, como Ecuador, por ejemplo, se analizan dos programas de I+D relacionados con la contratación pública y el fomento a la innovación, evidenciado que ninguno de estos instrumentos produce un efecto significativo en la decisión de las empresas de invertir en I+D (Fernández-Sastre \& Montalvo-Quizhpi, 2019).

Los efectos de la pandemia de COVID-19 dejaron ver ejemplos de negación y politización de la ciencia, al tiempo que desencadenaron el compromiso de los científicos frente a la producción de información con el objetivo de ayudar a los responsables políticos ante la problemática actual, cuyo impacto en países emergentes es más notorio, como indican Pardini et al. (2021). Con ello, se evidencia que el éxito de los programas e instrumentos de política pública no solo depende de la estabilidad política, sino también de recursos, planeación, evaluación y el correcto seguimiento a su implementación.

En el caso colombiano, a partir de la década de los noventa del siglo pasado, con la promulgación de la Ley 29 de 1990, se ha observado la preocupación por fomentar acciones que contribuyan al desarrollo de CTI, las cuales van desde el establecimiento de directrices, planes o estrategias - tales como, la transformación de Colciencias en el Departamento Administrativo, buscando fortalecer el Sistema Nacional de Ciencia, Tecnología e Innovaciónhasta la reciente decisión de crear un ministerio (Minciencias), por medio del cual Colciencias se convierte en el ente rector de la política de CTI nacional, cuyo objetivo es generar capacidades que promuevan el conocimiento científico y tecnológico, además de contribuir al desarrollo y el crecimiento del país (Ley 1951 de 2019). Así mismo, los planes de desarrollo planteados por el Gobierno Nacional han incorporado algunos lineamientos de política pública que señalan un relativo interés en esta materia, a pesar de que la inversión de recursos públicos y privados para ello aún se encuentra lejos de estándares internacionales, incluyendo a los países de la región. En este sentido, el documento de Plan Nacional de Desarrollo del país 2018-2022 (DNP, 2019a) plantea ejes o pactos estructurales (así como otros denominados transversales) dentro de los cuales se encuentra el de CTI. Por su parte, el Pacto por el emprendimiento, la formalización y la productividad, incluido en esta política, contempla entre sus estrategias a la transformación empresarial, el desarrollo productivo, la innovación y la adopción tecnológica para la productividad (DNP, 2019a, p. 120). 
En concordancia con lo anterior, el Plan Nacional de Desarrollo 2018-2022 contempla estrategias para fortalecer la institucionalidad, incrementar la productividad empresarial y mejorar la oferta de bienes públicos, con el fin de sofisticar el desarrollo productivo, incrementar apoyos a empresas para una modernización productiva y fortalecer la promoción de la investigación, el desarrollo y la innovación empresarial. En el marco de la política de desarrollo productivo, el documento Conpes 3866 de 2016 define el conjunto de instrumentos sectoriales y transversales para resolver las fallas de mercado, de gobierno y de articulación que inhiben el crecimiento de la productividad o que dificultan los procesos de sofisticación del aparato productivo nacional. Así, este documento propone como solución, entre otras, mejorar la capacidad de las unidades productoras para innovar y emprender, con lo cual se busca absorber y transferir conocimiento y tecnología en articulación con los actores del Sistema Nacional de Competitividad, Ciencia, Tecnología e Innovación (SNCTI). De manera particular, como un componente que retroalimenta las acciones de formación profesional, el Servicio Nacional de Aprendizaje (SENA) tradicionalmente ha llevado a cabo programas de innovación y desarrollo tecnológico, especialmente aquellos diseñados a partir de la expedición de la Ley 344 de 1996 (artículo 16).

Teniendo en cuenta que uno de los propósitos de las acciones señaladas es mejorar el bienestar general de la sociedad, las mediciones permiten poner en contexto el estado de las mismas, así como el balance entre el esfuerzo realizado y los resultados obtenidos. En este sentido, a nivel total de la economía, de acuerdo con cifras del Observatorio Colombiano de Ciencia y Tecnología, en las últimas dos décadas la financiación de actividades de ciencia tecnología e innovación (ACTI) en términos reales registró una tendencia creciente (figura 1), pasando de COP 1,06 billones en 2000 a 6,5 billones en 2019 (valores constantes de 2015). Como porcentaje del producto interno bruto (PIB), se observa un crecimiento más marcado entre 2012 y 2015 , explicado principalmente por los recursos provenientes del sistema de regalías, siendo el pico más alto en 2015 , cuando la inversión en este rubro representó $0,8 \%$ del PIB del país, seguido por una reducción en el ritmo de crecimiento hasta situarse en $0,74 \%$ en 2018 . Sin embargo, a pesar de este crecimiento, la inversión en ACTI aún se encuentra lejos del 1,5\% propuesto como meta para 2022 en el actual Plan Nacional de Desarrollo.

Frente al total de la inversión, en años recientes se observa una participación casi proporcional en la financiación de ACTI proveniente de recursos públicos y privados, hasta situarse en 2019 en 48 y $49 \%$, respectivamente, seguida de un $3 \%$ de recursos de origen internacional. En contraste con lo observado, de acuerdo con Zerda (2003), en los años noventa el mayor esfuerzo en la inversión en ciencia y tecnología correspondió de al sector público, debido principalmente a los créditos contratados con el Banco Interamericano de Desarrollo (BID).

Figura 1. Financiación de actividades ACTI en Colombia (2000-2019)

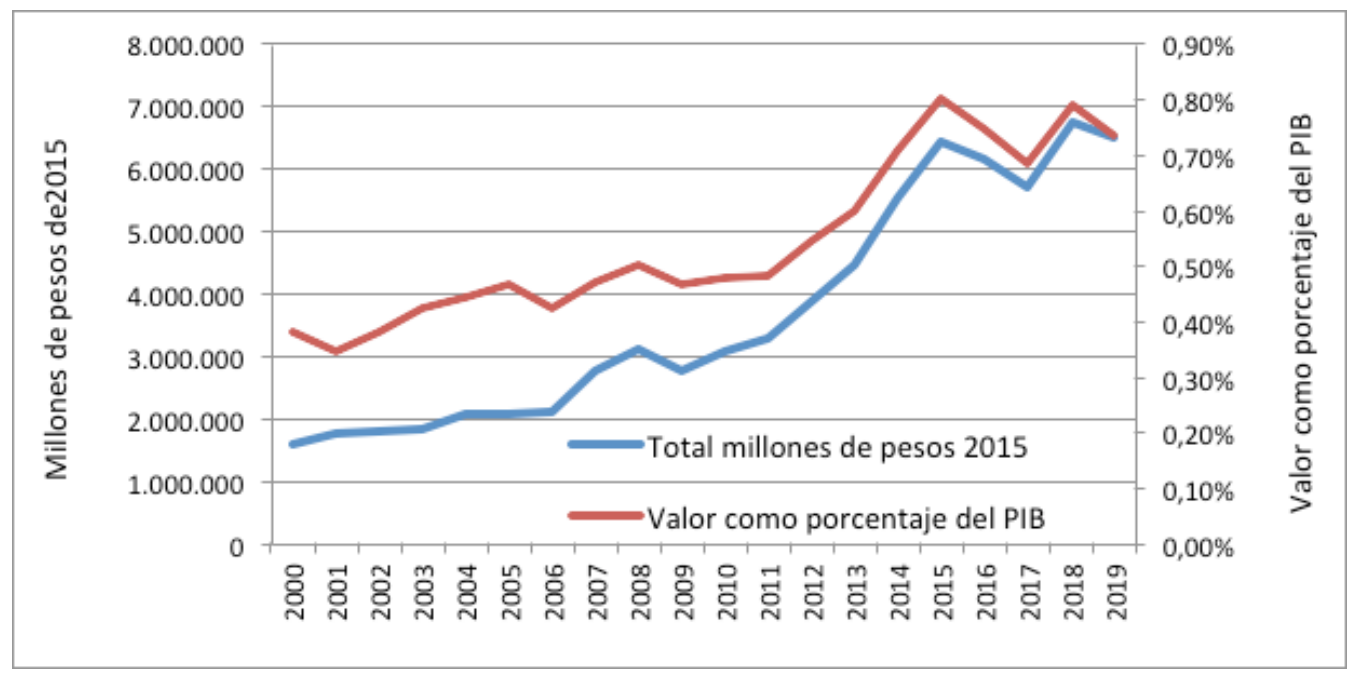

Fuente: Observatorio de Ciencia y Tecnología (2020) 
Teniendo en cuenta todo lo anteriormente mencionado, y considerando la importancia de las ACTI, este documento tiene como propósitos (i) realizar un balance de los desarrollos en materia de política pública en Colombia y algunos referentes internacionales, (ii) presentar un balance de las acciones que ha adelantado el SENA en esta materia y la incidencia de ellas sobre la formación profesional, y (iii) plantear algunos elementos que sirvan para la toma decisiones por parte de esta entidad. Teniendo en cuenta que, hasta donde sabemos, no se han realizado estudios particulares frente a inversión y resultados en acciones de investigación e innovación desde la formación para el trabajo, el presente estudio busca abordar esta temática mediante el empleo de fuentes primarias y secundarias, con el propósito de proporcionar nuevos referentes para el diseño de política pública.

El documento está organizado en cuatro secciones, siendo esta introducción la primera de ellas. A continuación, se presentan los aspectos metodológicos del trabajo. Posteriormente, se abordan los antecedentes teóricos y de política pública en torno a la innovación. En la tercera sección se reseñan elementos normativos y prácticos del diseño, ejecución y evaluación de la política nacional, particularmente en el SENA. Finalmente, se presentan las conclusiones y recomendaciones obtenidas tras la realización de esta investigación.

\section{ASPECTOS METODOLÓGICOS}

El Observatorio de Ciencia, Tecnología e Innovación (OCyT) y el Departamento Administrativo de Ciencia, Tecnología e Innovación adoptaron una metodología de medición de ACTI con base en los conceptos, términos y clasificaciones de referentes internacionales, así como la revisión de metodologías empleadas en el país (OCyT, 2008). En este sentido, se define a las actividades científicas y tecnológicas como aquellas que están estrechamente relacionadas con la producción, promoción, difusión y aplicación de los conocimientos científicos y técnicos en todos los campos de la ciencia y la tecnología. La tabla 1 resume los principales conceptos asociados.

Tabla 1. Clasificación de conceptos generales sobre ACTI

\begin{tabular}{|c|c|c|}
\hline Categoría & Descripción & Desagregación \\
\hline $\begin{array}{l}\text { Investigación y Desa- } \\
\text { rrollo Experimental } \\
(I+D)\end{array}$ & $\begin{array}{l}\text { Comprenden el trabajo creativo llevado a cabo de } \\
\text { forma sistemática para incrementar el volumen } \\
\text { de conocimientos humanos, culturales y sociales } \\
\text { y el uso de estos para derivar nuevas aplicaciones. }\end{array}$ & \\
\hline \multirow[t]{2}{*}{ Otras ACTI } & \multirow{2}{*}{$\begin{array}{l}\text { Comprenden la enseñanza y la formación cientí- } \\
\text { fica y técnica y los servicios científicos y técnicos. } \\
\text { Estos últimos incluyen, por ejemplo, actividades } \\
\text { de ciencia y tecnología, el control y la prospectiva, } \\
\text { la recogida de datos sobre fenómenos socioeco- } \\
\text { nómicos, los ensayos, la normalización y el control } \\
\text { de calidad, el asesoramiento a clientes y servicios, } \\
\text { y las actividades relacionadas con patentes y li- } \\
\text { cencias a cargo de las administraciones públicas. }\end{array}$} & $\begin{array}{l}\text { Apoyo a la formación y capacitación científica y tecno- } \\
\text { lógica: incluye los gastos en educación formal a nivel } \\
\text { de maestría y doctorado y la formación permanente de } \\
\text { científicos e ingenieros (postdoctorados y cursos de en- } \\
\text { trenamiento especializados). }\end{array}$ \\
\hline & & $\begin{array}{l}\text { Servicios científicos y tecnológicos: engloba las activida- } \\
\text { des relacionadas con la investigación y el desarrollo ex- } \\
\text { perimental que contribuyen a la producción, difusión y } \\
\text { aplicación de conocimientos científicos y técnicos. }\end{array}$ \\
\hline $\begin{array}{l}\text { Administración y } \\
\text { otras actividades de } \\
\text { apoyo }\end{array}$ & $\begin{array}{l}\text { Apoyan las actividades de I+D adelantadas por en- } \\
\text { tidades como ministerios, organismos de investi- } \\
\text { gación, fundaciones y otros organismos sin ánimo } \\
\text { de lucro mediante la recolección, administración y } \\
\text { distribución de fondos entre los ejecutores. }\end{array}$ & \\
\hline
\end{tabular}

M U T I S" | Revista electrónica editada por la Facultad de Ciencias Naturales e Ingeniería de UTADEO 


\begin{tabular}{|c|c|c|}
\hline Categoría & Descripción & Desagregación \\
\hline \multirow{6}{*}{$\begin{array}{l}\text { Actividades de inno- } \\
\text { vación }\end{array}$} & \multirow{6}{*}{$\begin{array}{l}\text { Todas aquellas etapas científicas, técnicas, co- } \\
\text { merciales y financieras que a pesar de no ser I+D } \\
\text { resultan necesarias para la puesta en marcha de } \\
\text { productos o servicios nuevos o mejorados y la } \\
\text { explotación comercial de procesos nuevos o me- } \\
\text { jorados. } \\
\text { Esta categoría incluye la adquisición de tecnolo- } \\
\text { gía (incorporada y no incorporada), el utilaje y la } \\
\text { ingeniería industrial, el diseño industrial, otras ad- } \\
\text { quisiciones de capital, el arranque del proceso de } \\
\text { fabricación y la comercialización de nuevos pro- } \\
\text { ductos. }\end{array}$} & $\begin{array}{l}\text { Diseño, instalación de maquinarias nuevas, ingeniería in- } \\
\text { dustrial y puesta en marcha de la producción. }\end{array}$ \\
\hline & & $\begin{array}{l}\text { Adquisición de maquinaria y equipos con desempeño } \\
\text { tecnológico mejorado (incluso software integrado) vin- } \\
\text { culados con las innovaciones implementadas por la em- } \\
\text { presa. }\end{array}$ \\
\hline & & $\begin{array}{l}\text { Adquisición de tecnología no incorporada al capital, ta- } \\
\text { les como patentes, inventos no patentados, licencias, } \\
\text { divulgaciones de know-how, diseños, marcas de fábrica } \\
\text { y patrones, así como de servicios de computación y otros } \\
\text { servicios científicos y técnicos relacionados con la imple- } \\
\text { mentación de innovaciones tecnológicas en productos y } \\
\text { procesos, además de la adquisición de paquetes de sof- } \\
\text { tware. }\end{array}$ \\
\hline & & $\begin{array}{l}\text { Modernización organizacional conducente a la introduc- } \\
\text { ción de cambios en el proceso productivo tendientes a } \\
\text { reducir tiempos muertos, desechos, tiempos de proce- } \\
\text { so u otros similares, todo ello con la línea de producción } \\
\text { existente. Modificaciones en el dibujo o diseño de la lí- } \\
\text { nea de producción (con las mismas máquinas y equipos } \\
\text { existentes), mejoras en la organización física de la planta } \\
\text { y desverticalización del proceso productivo, entre otros. }\end{array}$ \\
\hline & & $\begin{array}{l}\text { Actividades relacionadas con el lanzamiento de produc- } \\
\text { tos tecnológicamente nuevos o mejorados; entre ellas } \\
\text { se incluyen la investigación preliminar de mercado y la } \\
\text { publicidad de lanzamiento. }\end{array}$ \\
\hline & & $\begin{array}{l}\text { Capacitación en temas estrechamente relacionados con } \\
\text { las tecnologías centrales en el proceso productivo del } \\
\text { establecimiento. Pueden ser blandas (gestión y adminis- } \\
\text { tración) o duras (tecnología de procesos productivos), } \\
\text { e involucran un grado de complejidad significativo (no } \\
\text { evidente) que requiere de un personal capacitador alta- } \\
\text { mente especializado. }\end{array}$ \\
\hline
\end{tabular}

Fuente: elaboración propia con base en información del OCyT (2008).

Con base en esta clasificación se pretende identificar en qué medida las acciones realizadas por el SENA en materia de investigación, desarrollo tecnológico e innovación pueden ser consideradas como tal, cómo se ha dado su desarrollo en el tiempo, cuál ha sido el aporte de tales acciones, y si estas se encuentran alineadas con los desarrollos y las tendencias en materia de política pública.

\section{ASPECTOS CONCEPTUALES Y DE POLÍTICA PÚBLICA EN MATERIA DE CTI}

\section{Crecimiento económico y cambio técnico}

En la visión convencional de las teorías de crecimiento económico, el proceso de acumulación de capital físico 
y humano determina las diferencias de productividad entre países, siendo el cambio en la tecnología (entendido como el logro más eficiente de la combinación de factores de producción para la obtención del producto) el único factor que explica las modificaciones en las tasas de crecimiento. Si bien se han presentado divergencias y discusiones en torno a aspectos como el papel del ahorro, la inversión o la caracterización de un agente representativo, en general, se mantiene el cambio tecnológico como factor fundamental del crecimiento económico de las naciones. Esta visión hace referencia a los modelos de Solow, Romer, Aghion y Howitt, planteados y revisados en la segunda mitad del siglo XX. A juicio de Hernández (2004), la principal debilidad de estas concepciones es el empleo de funciones de producción representativas, lo cual desconoce las implicaciones teóricas y de política existentes en las diferentes instituciones y organizaciones.

Una segunda vertiente de pensamiento se ha concentrado en indagar por las formas específicas en que se genera el cambio técnico y cómo este se difunde, o cómo se producen procesos de interacción entre los diferentes agentes. Frente a ello, Schumpeter introduce el concepto de "destrucción creadora" al considerar que los motores del desarrollo económico (concepto más amplio que crecimiento económico) son la tecnológica y las fuerzas socioculturales, fenómenos capaces de provocar transformaciones decisivas en la sociedad y la economía (Montoya, 2004).

De las corrientes teóricas reseñadas, en esencia, se desprende el papel importante que juegan la ciencia y la tecnología en la transformación del aparato productivo y las relaciones sociales. Al respecto, se observa que en muchos países las políticas públicas orientadas a la ciencia y la tecnología ocupan un lugar superior en el diseño de estrategias para el desarrollo frente a la formulación de otro tipo de acciones focalizadas, como la política industrial (Zerda, 2003).

El diseño de política en ciencia y tecnología generalmente incluye acciones de investigación y desarrollo $(I+D)$, las cuales son consideradas de gran valor para la creación de nuevo conocimiento y son precursoras del desarrollo. No obstante, estas actividades deben incluir la aplicación práctica de avances a través de la innovación, en el marco de una institucionalidad que permita la participación de diversos agentes. Este ciclo es denominado por Fuentes y Arguimbau (2008) como el sistema completo de la investigación (I+D+i).
De una manera más esquemática, Michel Callón planteó este ciclo o proceso de creación, difusión y uso de las innovaciones mediante el diagrama denominado "rosa de los vientos de la investigación", en el cual los diferentes agentes que participan (directa o indirectamente) pueden ser agrupados en cinco polos, como se muestra en la figura 2.

Figura 2. Proceso de creación, difusión y uso de innovaciones

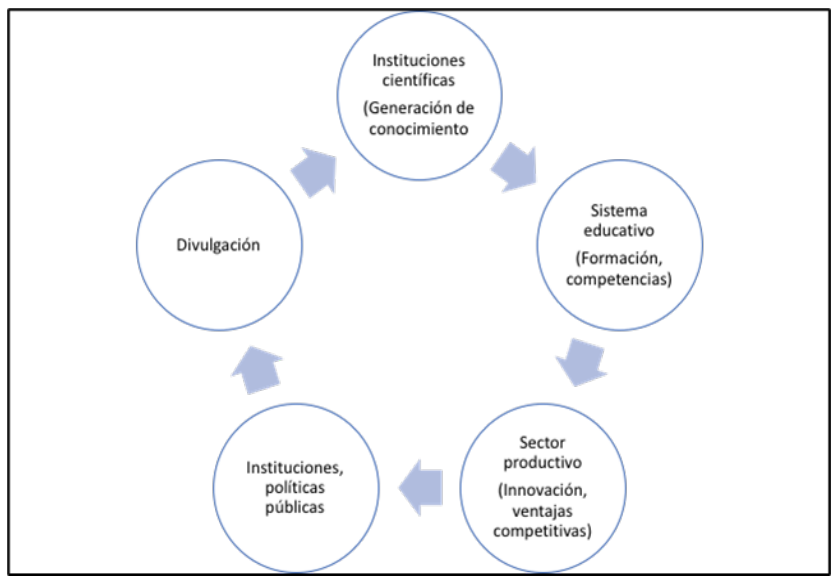

Fuente: elaboración propia con base en Pujol y Arguimbau (2008, p. 49).

Este sistema plantea diferentes niveles y grados de participación de los agentes en la generación o difusión de conocimiento. Sin embargo, vale la pena señalar dos factores determinantes para el logro de su fin último, es decir, potenciar el desarrollo económico y la competitividad empresarial. En primer lugar, este tipo de sistemas debe contar con los incentivos adecuados para fomentar la coordinación y convergencia entre agentes para el logro de propósitos comunes, aspecto que usualmente se busca con la conformación o implementación (no necesariamente formal) de los sistemas de CTI. En segundo lugar, se plantea la necesidad de analizar y evaluar periódicamente el proceso con el fin de tomar decisiones de política pública, temas que se abordan en el siguiente numeral.

\section{Diseño y ejecución de política pública en la región}

De acuerdo con Pagés (2010), la principal razón del modesto crecimiento económico de Latinoamérica es la baja productividad de los países de la región. Esta autora precisa que mientras en 1960 el ingreso per cápita de la región representaba una cuarta parte del ingreso de Estados Unidos, para 2005 esta proporción 
equivalía a una sexta parte (Pagés, 2010, p. 10). Por lo tanto, se plantea la necesidad de diseñar políticas ambiciosas para el uso adecuado de los recursos existentes en la región, es decir, más eficiencia de la mano de obra, el capital físico y humano y el despliegue de tecnologías que permitan reducir la brecha de ingreso de Latinoamérica frente al resto del mundo. A partir de ello, en materia de política pública de fomento a la ciencia, la tecnología y la innovación, se puede apreciar tres enfoques generacionales en los últimos cuatro decenios, los cuales se detallan en la figura 3.

Figura 3. Generalidades de políticas públicas en materia de CTI en Latinoamérica

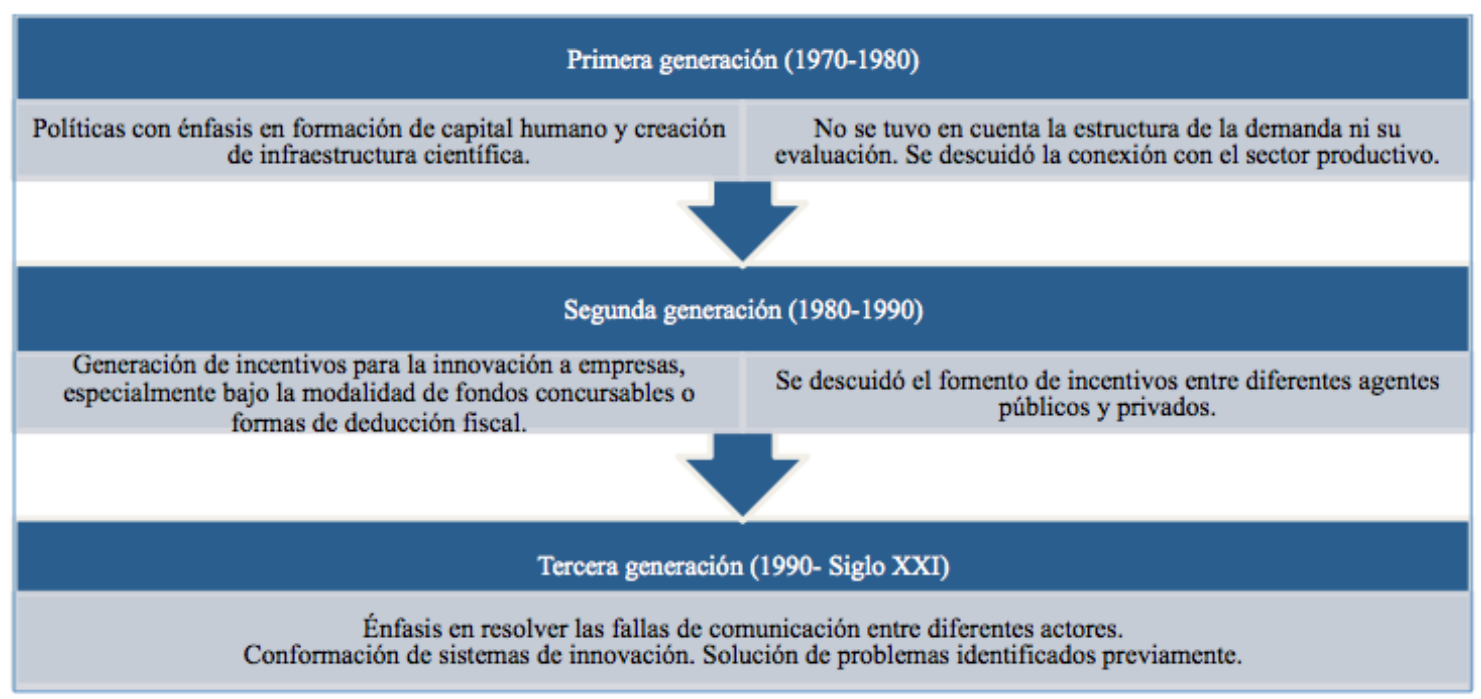

Fuente: elaboración propia con base en Pagés (2010).

En la figura 3 se observa cómo la política pública en CTI en Latinoamérica evolucionó desde los programas focalizados a la formación científica - un poco desconectados de las necesidades de las naciones - hasta el diseño de sistemas de CTI con énfasis en la generación de incentivos adecuados para facilitar la integración y convergencia de intereses entre diferentes agentes. La evolución mediante el cambio de enfoques no implica que el último sea incompatible con los anteriores, sino que se dio una reorientación para focalizar ciertas acciones que permitieran incrementar el potencial de innovación de la región.

A partir de la experiencia en el diseño de política en la región, se evidencia que el fomento del cambio tecnológico es fundamental. Sin embargo, no se debe descuidar la alineación de incentivos entre empresas, gobierno e instituciones, así como el diseño de mecanismos efectivos que garanticen oportunidades y acceso justo a los recursos. Sobre el tema, la evidencia señala que las empresas de la región que más invierten en innovación no son las de mayor tamaño, las más exportadoras o aquellas con mayor capital extranjero, sino que son las empresas con acceso a financiamiento, protección eficaz de la propiedad intelectual y aquellas que cuentan con cooperación tecnológica con clientes, proveedores o entidades que intervienen en la transmisión de conocimiento aplicado (Pagés, 2010). Por ejemplo, Navarro et al. (2010), quienes emplearon un método cuantitativo para estimar la tendencia a invertir en innovación en Latinoamérica, dan cuenta de la relación positiva del financiamiento público para la innovación con la protección formal de la propiedad intelectual y la cooperación tecnológica entre empresas (proveedores y clientes), laboratorios y universidades (p. 270). Otros instrumentos observados por Navarro et al. (2010) son reseñados en la tabla 2 . 
Tabla 2. Instrumentos de política para fomentar la innovación en Latinoamérica (países seleccionados)

\begin{tabular}{|c|c|c|c|c|c|c|c|c|c|c|c|c|c|}
\hline Instrumento & 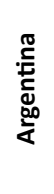 & $\begin{array}{l}\overline{\bar{n}} \\
\overline{5} \\
\bar{\infty}\end{array}$ & 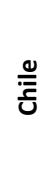 & $\begin{array}{l}\frac{0}{0} \\
\frac{0}{\varepsilon} \\
\frac{0}{0}\end{array}$ & 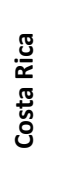 & $\begin{array}{l}\frac{2}{0} \\
\frac{\pi}{\pi} \\
\frac{2}{\pi} \\
\frac{\pi}{4}\end{array}$ & 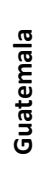 & $\underset{\substack{: \\
:}}{\stackrel{0}{x}}$ & 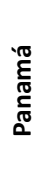 & 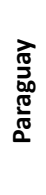 & : & 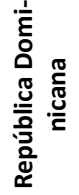 & 贾 \\
\hline \multicolumn{14}{|c|}{ Primera generación } \\
\hline $\begin{array}{l}\text { Fondo de investigación científica y } \\
\text { tecnológica }\end{array}$ & $x$ & $\mathrm{x}$ & $x$ & $x$ & $x$ & & $x$ & $x$ & $x$ & $x$ & $x$ & $x$ & $x$ \\
\hline $\begin{array}{l}\text { Apoyo a la creación de centros de } \\
\text { excelencia }\end{array}$ & $x$ & $x$ & $x$ & $x$ & & & & $x$ & & & & & $x$ \\
\hline $\begin{array}{l}\text { Becas para graduados y profesiona- } \\
\text { les con posgrado }\end{array}$ & $x$ & $x$ & $x$ & $x$ & & & & $x$ & $x$ & & $x$ & & $x$ \\
\hline $\begin{array}{l}\text { Programas de apoyo a ciudadanos } \\
\text { con estudios de posgrado en ciencia } \\
\text { y tecnología }\end{array}$ & $\mathrm{x}$ & $\mathrm{X}$ & $x$ & $x$ & & & & $\mathrm{x}$ & $x$ & $x$ & & & $x$ \\
\hline $\begin{array}{l}\text { Incentivos salariales para la investi- } \\
\text { gación en ciencia y tecnología }\end{array}$ & $x$ & & & & & & & $x$ & $x$ & & & & $x$ \\
\hline $\begin{array}{l}\text { Fortalecimiento de nexos con inves- } \\
\text { tigadores nacionales que trabajan en } \\
\text { el extranjero }\end{array}$ & $x$ & & $x$ & & & & & $x$ & $x$ & & & & $x$ \\
\hline \multicolumn{14}{|c|}{ Segunda generación } \\
\hline $\begin{array}{l}\text { Fondos para promover la innovación } \\
\text { y la competitividad en las empresas }\end{array}$ & $x$ & $x$ & $x$ & $x$ & $x$ & $x$ & & $x$ & $x$ & $x$ & $x$ & & $x$ \\
\hline $\begin{array}{l}\text { Capital emprendedor, capital genera- } \\
\text { dor y otros instrumentos financieros } \\
\text { para la innovación }\end{array}$ & $x$ & $x$ & $x$ & $x$ & & & & & & & & & $x$ \\
\hline $\begin{array}{l}\text { Incentivos fiscales para I+D e inno- } \\
\text { vación }\end{array}$ & $x$ & $x$ & $x$ & $x$ & & & & $x$ & & & & & \\
\hline $\begin{array}{l}\text { Mecanismos para promover la } \\
\text { transferencia de tecnología y cono- } \\
\text { cimiento al sector no agropecuario } \\
\text { (extensión tecnológica, etc.) }\end{array}$ & $x$ & & & & & & & $\mathrm{x}$ & $x$ & $x$ & & & \\
\hline \multicolumn{14}{|c|}{ Tercera generación } \\
\hline $\begin{array}{l}\text { Fondos de innovación para sectores } \\
\text { seleccionados con alto potencial } \\
\text { para innovar }\end{array}$ & $x$ & $x$ & $\mathrm{x}$ & & & & & $x$ & & & & & $x$ \\
\hline Áreas / sectores prioritarios & $x$ & $x$ & $x$ & $x$ & & & & $x$ & & $x$ & & & $x$ \\
\hline $\begin{array}{l}\text { Promoción de polos de tecnología e } \\
\text { incubadoras de negocios }\end{array}$ & $x$ & $x$ & $x$ & & & & & & & & & $x$ & $x$ \\
\hline $\begin{array}{l}\text { Otros instrumentos para mejorar los } \\
\text { sistemas regionales de innovación }\end{array}$ & & $x$ & $x$ & & & & & $x$ & & & & & \\
\hline $\begin{array}{l}\text { Mecanismos de diálogo entre } \\
\text { actores de los sistemas nacionales } \\
\text { de innovación (tablas de tecnología } \\
\text { e innovación, etc.) }\end{array}$ & $x$ & $x$ & $x$ & & & & & $x$ & & & & & $x$ \\
\hline
\end{tabular}

Fuente: Navarro et al. (2010). 
Con respecto a la inversión pública en ACTI, es preciso señalar que en Latinoamérica esta es inferior a 1 $\%$ del PIB de cada nación, en tanto que en otros países de la OCDE esta alcanza 2,34\%. Una característica particular de la región es que la inversión tiene un componente importante en la adquisición de equipos y bienes de capital (entre 50 y $80 \%$ ), lo cual se considera excesivo, toda vez que, si bien contribuye a cerrar la brecha tecnológica, su resultado depende de las capacidades internas en I+D. En contraste, la inversión del sector privado en innovación es particularmente alta. Por ejemplo, en Colombia, $46 \%$ de las grandes empresas innovan, mientras que en países como Chile esta cifra llega a $30 \%$. No obstante, en Colombia la inversión en temas de innovación es baja en comparación con el gasto en I+D como porcentaje del PIB, permaneciendo estable en torno a $0,25 \%$, cifra que se ubica por debajo de otros países de la región, como Brasil (1,2\%), el país con mayor inversión, Chile (0,39\%), México y Argentina (ambos alrededor de $0,5 \%$ ).

Frente al impacto de dichas inversiones, a pesar de las deficiencias y características de la inversión en innovación en Latinoamérica, algunos estudios señalan un resultado positivo sobre la productividad, aunque más en personas que en procesos, lo que algunos casos depende de las características de las empresas. En contraste, no se observan efectos significativos de las inversiones en innovación sobre la organización, o en otros casos se evidencia un efecto tardío.

Una posible explicación a los modestos resultados de las inversiones en innovación por parte del sector productivo es que los procesos de aprendizaje y adopción de nuevos métodos en las economías latinoamericanas toman más tiempo debido a la capacidad del capital humano, desincentivos para invertir decididamente en innovación y desconocimiento de los procesos de propiedad intelectual, entre otros.

Como parte de un análisis más reciente para la región, la UNESCO (2018) señala la necesidad de vincular la investigación endógena con la innovación en el sector productivo, para lo cual son necesarias políticas sectoriales de largo plazo. Si bien en años recientes se observan algunas tendencias en este sentido (tabla 3), aún se presentan resultados variables entre países.

En este contexto, la UNESCO señala que para la reducción de la brecha entre países resulta importante aumentar el número de investigadores, así como realizar una mayor inversión en enseñanza superior, producción científica y colaboración científica internacional. Este último aspecto cobra relevancia en la coyuntura asociada con la emergencia sanitaria derivada del COVID-19, la cual ha movilizado acciones y recursos entre países e instituciones para afrontar los retos en CTI (BID, 2020). Se tiene entonces que un aspecto fundamental para el avance hacia un sistema de CTI de tercera generación, que genere incentivos adecuados y sinergias entre los diferentes agentes que lo integran, es la formación de capital humano articulado con proyectos y necesidades del sector real. En particular, esta variable se asocia con la invención en innovaciones radicales, así como la adaptación que implica la innovación incremental. Desde una perspectiva complementaria, Ibáñez (2018) realiza un análisis estadístico de variables para Latinoamérica, encontrando una relación positiva entre la extensión de un país (superficie, población, PIB, número de investigadores y número de publicaciones científicas de excelencia) y su relevancia en el contexto internacional en investigación y desarrollo tecnológico.

Con relación al efecto en el crecimiento económico, en varios países de Latinoamérica este ha sido asociado al crecimiento del capital físico y humano, y en menor medida a la productividad, lo cual se relaciona con un bajo grado de innovación en las empresas. En Colombia, al igual que en los demás países de la región, a pesar de contar con el capital humano y físico necesario, la productividad y los procesos de innovación en las empresas son bajos, lo cual se debe a que el capital humano vinculado al sector laboral no cuenta con las capacidades idóneas para innovar dentro de las empresas (figura 4). 
Tabla 3. Inventario de instrumentos de política de CTI en Latinoamérica, 2010-2015 (países seleccionados)

\begin{tabular}{|c|c|c|c|c|c|c|c|c|c|c|c|c|c|}
\hline$\frac{n}{\sigma 0}$ & 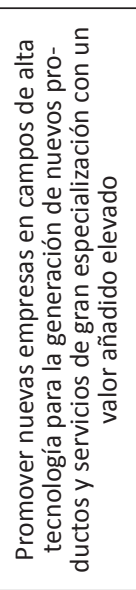 & 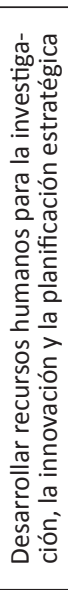 & 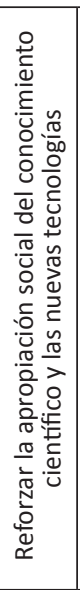 & 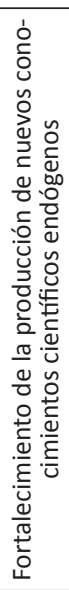 & 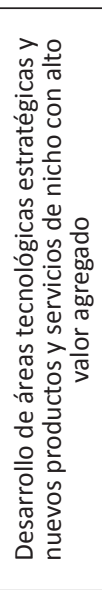 & 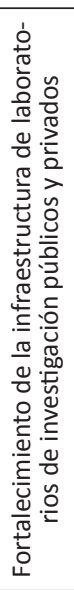 & 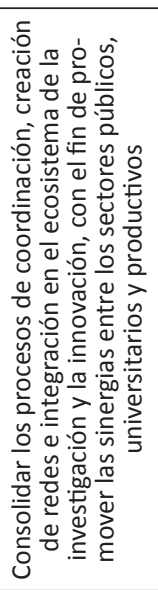 & 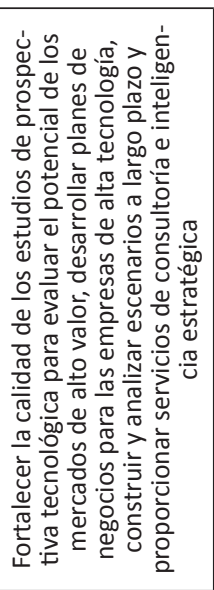 & 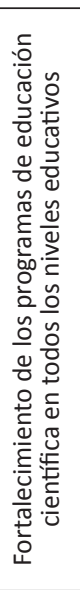 & 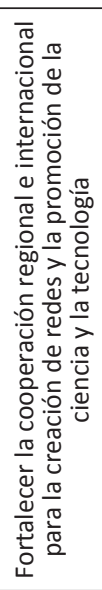 & 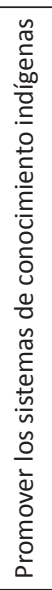 & 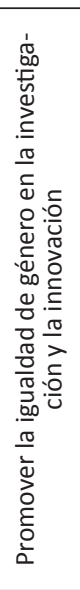 & 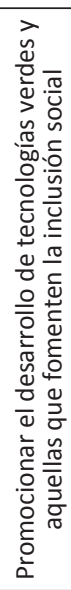 \\
\hline Chile & 37 & 25 & 24 & 25 & 17 & 12 & 6 & 14 & 7 & 6 & & 6 & \\
\hline Argentina & 38 & 25 & 32 & 22 & 15 & 9 & 14 & 12 & 5 & 10 & 5 & 2 & 4 \\
\hline Brasil & 27 & 31 & 6 & 15 & 15 & 10 & 5 & 8 & 5 & 4 & & 6 & 5 \\
\hline México & 19 & 13 & 6 & 16 & 14 & 9 & 4 & 6 & 6 & 5 & 3 & 5 & \\
\hline Uruguay & 14 & 11 & 13 & 13 & 9 & 3 & 3 & 8 & 2 & 4 & & 1 & 3 \\
\hline El Salvador & 2 & 2 & 5 & & & 4 & & 6 & 9 & & & & 1 \\
\hline Perú & 6 & 12 & 6 & 10 & 3 & 7 & & 1 & 5 & 2 & 1 & 1 & \\
\hline Paraguay & 3 & 6 & 5 & 8 & 4 & 1 & 3 & 2 & 1 & 5 & & & \\
\hline Costa Rica & 4 & 10 & 23 & 2 & 4 & 2 & & 4 & 3 & 4 & & 2 & \\
\hline Venezuela & 2 & 3 & 7 & 5 & & 1 & & 2 & & 1 & & 2 & \\
\hline Panamá & 4 & 14 & 6 & 5 & & 2 & 1 & 1 & 3 & 1 & & & \\
\hline Colombia & 6 & 2 & 10 & 6 & 1 & 1 & 2 & 2 & & 1 & 3 & 1 & 1 \\
\hline Ecuador & 4 & 5 & 4 & & 2 & & 1 & 1 & 2 & & 4 & & \\
\hline Guatemala & 4 & 6 & 6 & 3 & & & & 1 & 2 & & & & \\
\hline Bolivia & 5 & 1 & 8 & 2 & 1 & 1 & & 3 & 1 & 1 & 4 & 1 & 1 \\
\hline Honduras & 1 & 1 & 1 & 1 & & & & & 2 & & & & \\
\hline Nicaragua & & & & 1 & & 1 & & & & 1 & & & \\
\hline Cuba & & & 5 & & & & & 1 & & & & & \\
\hline $\begin{array}{l}\text { República } \\
\text { Dominicana }\end{array}$ & & & 1 & & & & & & & & & & \\
\hline
\end{tabular}

Fuente: Unesco (2018).

Figura 4. Participación de factores en el crecimiento promedio (2000-2016)

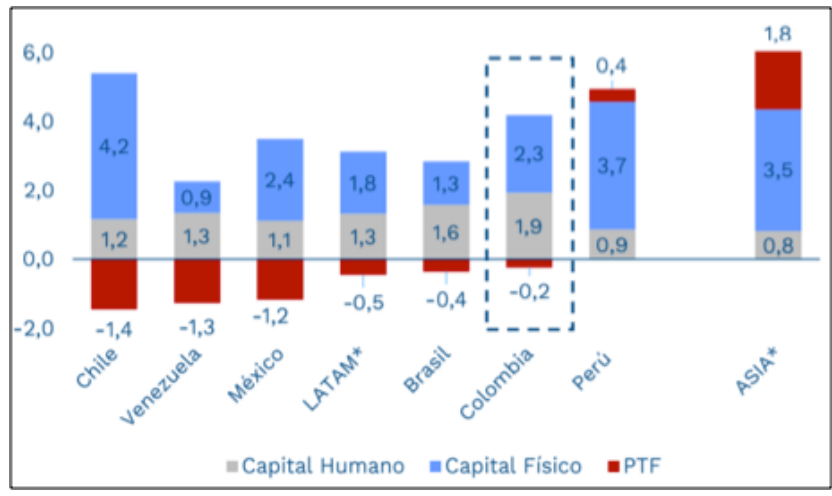

Fuente: DNP (2019b).
Ante este escenario, cobra relevancia la contribución de la ciencia, la tecnología y la innovación a la formación para el trabajo, dinámica que busca generar capacidades en el recurso humano para fortalecer los procesos de productividad y competitividad en las empresas. Al respecto, la Oficina Regional de la Organización Internacional del Trabajo (OIT) para América Latina y el Caribe y el Centro Interamericano para el Desarrollo del Conocimiento de la Formación Profesional (OIT/Cinterfor) (2017) destacan al SENA como referente, siendo muy cercano a la experiencia del Servicio Nacional de Aprendizaje Industrial (SENAI) en Brasil, que en la década de los años setenta 
estableció los centros de desarrollo tecnológico con el objetivo de prestar servicios en aspectos tales como asistencia técnica, asesoría, consultoría, diseño, investigación aplicada, pruebas y ensayos de laboratorio, entre otros. Posteriormente, el SENA diversificó su campo de acción a través del Sistema de Investigación, Desarrollo Tecnológico e Innovación SENNOVA, el cual, al igual que el caso de Brasil, fortalece la idea de que la formación profesional debe apoyar la innovación y el desarrollo de capacidades productivas.

Cinterfor (2017) señala un cambio de paradigma a partir de las últimas décadas del siglo veinte, en el que la tendencia de los centros de formación a escala global busca que el desarrollo de competencias y habilidades del recurso humano esté mediado por la incorporación del diseño, la investigación y la innovación en los procesos formativos, proceso donde el SENA cuenta con una importante trayectoria en política de investigación, desarrollo tecnológico e innovación. En el caso particular del SENA, Lugo (2017) analiza los aportes de la formación profesional a los procesos de innovación, encontrando un vínculo que permite acelerar la innovación empresarial con base en la transferencia de conocimiento, la actualización tecnológica y la participación de la fuerza laboral de los trabajadores.

\section{Evaluación de programas y diseño de una política que propende por su articulación}

En el presente siglo se han llevado a cabo diferentes evaluaciones a programas o instrumentos ejecutados por entidades gubernamentales con base en el SCTI. En general, los resultados son positivos, aunque también variados, dependiendo de los objetivos y el alcance de cada iniciativa. Entre los estudios orientados a evaluar el diseño, la metodología o el impacto de programas institucionales en períodos específicos, se encuentran los trabajos realizados por SantaMaría et al. (2009), Tecnos (2010), la Universidad del Norte y la Universidad Industrial de Santander (2010), el Centro de Investigaciones para el Desarrollo (CID, 2010), Maffioli y Meléndez (2011) y Econometría Consultores et al. (2014).

Estudios más recientes se han enfocado en analizar tanto aspectos particulares como al sistema en su conjunto. Por ejemplo, un trabajo iniciado por el Banco Mundial en 2015 (Cruz et al., 2019) analiza el gasto público en CTI a partir de la identificación de 129 instrumentos pertenecientes a 8 instituciones del Gobierno Nacional, con el objetivo de evaluar la composición y coherencia de los instrumentos de la política, así como la calidad de los procesos, los sistemas y la funcionalidad de la política. Entre los principales resultados se encuentra (i) la identificación de una alta concentración del presupuesto en pocos instrumentos a nivel agregado y también a nivel de cada institución; (ii) la identificación de un grado significativo de superposición en instrumentos que tienen el mismo objetivo o beneficiario; (iii) la falta de suficientes instrumentos para la atención de empresas en estado inicial (startups), lo que tiene implicaciones negativas en la consecución del objetivo de diversificación económica; (iv) evidencia de que el desempeño promedio de las dimensiones de implementación y gobernanza obtuvo un mejor puntaje que las dimensiones de diseño; ( $v$ ) la concentración pronunciada de los instrumentos de CTI en un número muy reducido de mecanismos de intervención (mayoritariamente subsidios); y (vi) una heterogeneidad general en el desempeño funcional dentro de cada institución.

Con base en los resultados de la primera fase del mencionado estudio, se introdujeron cambios en algunos programas en cabeza de algunos ministerios y el SENA, llegando a formular acciones que fueron contempladas en el documento Conpes 3866, Política Nacional de Desarrollo Productivo (PDP). En la segunda fase del estudio se buscó identificar la forma en que los programas implementados convierten insumos en productos y resultados esperados, a partir de un ejercicio piloto a 5 programas, a saber, Beneficios Tributarios (Colciencias, hoy Minciencias) (Minciencias, 2020a), Becas de Doctorado Colciencias (Minciencias, 2020b), Capital Semilla (iNNpulsa) (iNNpulsa, 2021), Apps.co (Ministerio de Tecnologías de Información y Comunicación TIC) (MinTIC, 2021) y Desarrollo Tecnológico (Servicio Nacional de Aprendizaje) (SENA, 2021).

Con dicho diagnóstico, el Gobierno Nacional se encuentra en el proceso de implementación de una metodología denominada "Articulación para la Competitividad, Arco" (Directiva Presidencial n. 12 del 18 de diciembre de 2019 y circular externa del Departamento Nacional de Planeación n. ${ }^{\circ}$ 02-4 del 16 de enero de 2020) (SNCl, 2021), cuyo propósito es evaluar y definir lineamientos orientados a mejorar la eficacia de instrumentos de política en materia de competitividad, productividad, emprendimiento, 
ciencia, tecnología e innovación en las diferentes instituciones que ejecutan programas y proyectos de este tipo. El resultado esperado se centra en la articulación de entidades e instrumentos para el desarrollo de herramientas estándar, la reducción de la atomización de recursos y la obtención de mejoras en funcionalidad e impacto. En general, se observa el interés por fortalecer los diferentes aspectos que contribuyen a la consolidación de un sistema que opere de manera articulada entre los diferentes actores.

\section{POLÍTICA DE INNOVACIÓN Y DESARROLLO TECNOLÓGICO Y FORMACIÓN PARA EL TRABAJO}

\section{Normatividad colombiana en CTI}

En Colombia, a partir de la década de 1990 se observa un paulatino desarrollo de normas tendientes a fomentar condiciones que permitan crear programas y construir institucionalidad en torno a políticas de CTI. Se observa entonces que la Constitución Política de 1991 le asigna al Estado colombiano la obligación de promover la investigación, la ciencia, el desarrollo y la difusión de los valores culturales de la nación (Artículo 70, reglamentado por la Ley 1675 de 2013). De igual manera, mediante la Ley 29 de 1990, "por la cual se dictan disposiciones para el fomento de la investigación científica y el desarrollo tecnológico y se otorgan facultades extraordinarias", se le asignó al Estado la promoción y orientación en torno a los adelantos científicos y tecnológicos, en articulación con la universidad, la comunidad científica y el sector privado, lo cual debe plasmarse en los planes de desarrollo. Esta norma y sus decretos reglamentarios facultan a las entidades públicas para la celebración de contratos y convenios que faciliten, fomenten o desarrollen actividades científicas y tecnológicas. En igual sentido, los decretos 393 y 585 de 1991 regulan las modalidades específicas de contratos de fomento de actividades científicas y tecnológicas entre particulares y entidades públicas. Posteriormente, la Ley 1286 de 2009 estableció que los contratos y convenios que se celebren con el objeto de promover la ciencia y la tecnología se encuentran regulados en sus aspectos sustantivos por las normas especiales de los decretos 393 y 591 de 1991; sin embargo, estos se encuentran sujetos a la Ley 80 de 1993 en todo lo no regulado por aquellas normas con fuerza legal.
En relación con la formación profesional para el trabajo, el SENA tiene entre sus funciones participar en actividades de investigación y desarrollo tecnológico, ocupacional y social que contribuyan a la actualización y el mejoramiento de la formación profesional integral (Artículo 3 de la Ley 119 de 1994). Adicionalmente, esta entidad destina parte de sus ingresos al desarrollo de programas de competitividad y productividad como parte de la contribución del Estado al SNCTI. De esta manera, el Consejo Directivo Nacional del SENA, a través de varios acuerdos, ha reglamentado los diferentes programas que contribuyen a la competitividad, el desarrollo tecnológico y la formación profesional. De igual manera, existen políticas y normas de política pública a las cuales el SENA se adhiere para el diseño de sus acciones, entre las que se incluyen el Plan Nacional de Desarrollo y las leyes y los documentos del Consejo Nacional de Política Económica y Social (Conpes) en materia de $\mathrm{CTI}$, productividad y competitividad. Sumado a lo anterior, recientemente fue expedida la Ley 1951 de 2019, por la cual se crea el Ministerio de Ciencia, Tecnología e Innovación (Minciencias), ente rector de la política pública nacional en CTI, y se busca fortalecer el SNCTI.

Teniendo en cuenta el actual escenario, se observa que de manera paulatina se ha buscado la elaboración de normas que permitan crear incentivos para el funcionamiento de un sistema que integre los diferentes actores y además articule acciones que contribuyan el desarrollo científico y tecnológico del país.

\section{Política de investigación, desarrollo tecnológico e innovación del SENA, SENNOVA}

Desde la promulgación de la Ley 344 de 1996, en particular su artículo 16, el Consejo Directivo Nacional del SENA ha expedido acuerdos y líneas programáticas para el desarrollo de acciones de desarrollo tecnológico y competitividad, los cuales entre 1996 y 2013 fueron coordinados por diferentes áreas de la Entidad, de conformidad con los objetivos misionales de las mismas. La tabla 4 describe los principales programas enfocados a ACTI (OCYT, 2008).

De otro lado, en cuanto al manejo de recursos, entre 2002 y 2013 el SENA ejecutó COP 2,01 billones en las diferentes líneas señaladas, de conformidad con lo detallado en la tabla 5. 
Tabla 4. Programas de competitividad y desarrollo tecnológico desarrollados por el SENA (1996-2013)

\begin{tabular}{|c|c|c|c|}
\hline Área & Programa & Objetivo & $\begin{array}{l}\text { Clasifica- } \\
\text { ción ACTI }\end{array}$ \\
\hline $\begin{array}{l}\text { Dirección de Formación Profesio- } \\
\text { nal, Dirección del Sistema Nacional } \\
\text { de Formación para el Trabajo, } \\
\text { Dirección de Planeación y Direccio- } \\
\text { namiento Corporativo }\end{array}$ & $\begin{array}{l}\text { Innovación y desa- } \\
\text { rrollo tecnológico } \\
\text { productivo }\end{array}$ & $\begin{array}{l}\text { En alianza con entidades vinculadas al SNCTI, busca cofi- } \\
\text { nanciar proyectos de empresas con el propósito de contri- } \\
\text { buir al desarrollo tecnológico del país y a la implementa- } \\
\text { ción de procesos innovativos en el sector productivo. }\end{array}$ & $\begin{array}{l}\text { Activida- } \\
\text { des de } \\
\text { innovación }\end{array}$ \\
\hline $\begin{array}{l}\text { Dirección de Formación Profesio- } \\
\text { nal, Dirección del Sistema Nacional } \\
\text { de Formación para el Trabajo, } \\
\text { Dirección de Planeación y Direccio- } \\
\text { namiento Corporativo }\end{array}$ & $\begin{array}{l}\text { Alianza SENA - Col- } \\
\text { ciencias }\end{array}$ & $\begin{array}{l}\text { Financiar actividades que promueven y fomentan la inves- } \\
\text { tigación aplicada, la innovación, el desarrollo tecnológico, } \\
\text { la apropiación técnica de la ciencia, la tecnología y, en } \\
\text { general, la construcción de capacidades regionales de } \\
\text { ciencia, tecnología e innovación. }\end{array}$ & $\begin{array}{l}\text { Adminis- } \\
\text { tración } \\
\text { y otras } \\
\text { actividades } \\
\text { de apoyo }\end{array}$ \\
\hline Dirección de Formación Profesional & $\begin{array}{l}\text { Modernización de la } \\
\text { oferta de formación } \\
\text { profesional en Cen- } \\
\text { tros de Formación }\end{array}$ & $\begin{array}{l}\text { Modernizar y actualizar los Centros de Formación Pro- } \\
\text { fesional del SENA mediante la adaptación de las nuevas } \\
\text { tecnologías, para ofrecer formación profesional por com- } \\
\text { petencias laborales en los diferentes sectores productivos } \\
\text { y con la estrategia de ambientes móviles. }\end{array}$ & $\begin{array}{l}\text { Activida- } \\
\text { des de } \\
\text { innovación }\end{array}$ \\
\hline Dirección de Formación Profesional & Parques tecnológicos & $\begin{array}{l}\text { Consolidar una red para promover talento hacia el } \\
\text { desarrollo tecnológico, la innovación y el emprendimien- } \\
\text { to. Disponer herramientas, infraestructura y asesoría } \\
\text { de expertos en un ambiente que permita la apropiación } \\
\text { tecnológica para el desarrollo de nuevas iniciativas de } \\
\text { productos y servicios, en líneas tales como biotecnología, } \\
\text { agroindustria, nanotecnología, electrónica y telecomunica- } \\
\text { ciones, tecnologías virtuales, diseño e ingeniería. }\end{array}$ & $\begin{array}{l}\text { Activida- } \\
\text { des de } \\
\text { innovación }\end{array}$ \\
\hline Oficina de Sistemas & SENA Digital & $\begin{array}{l}\text { Fortalecer e implementar nuevos servicios de tecnologías } \\
\text { de la información y las comunicaciones (TIC), buscando } \\
\text { contribuir a la provisión de información adecuada para la } \\
\text { gestión de la entidad y garantizar el acceso de la población } \\
\text { colombiana a las diferentes fuentes de conocimiento, } \\
\text { los ambientes virtuales de formación y las plataformas } \\
\text { colaborativas como elemento clave en la formación para } \\
\text { el trabajo, la innovación y el emprendimiento. }\end{array}$ & $\begin{array}{l}\text { Activida- } \\
\text { des de } \\
\text { innovación }\end{array}$ \\
\hline $\begin{array}{l}\text { Dirección de Promoción y Relacio- } \\
\text { nes Corporativas }\end{array}$ & Internacionalización & $\begin{array}{l}\text { Apoyar el desarrollo de proyectos de cooperación interna- } \\
\text { cional que permitan la transferencia de conocimientos y } \\
\text { tecnologías al SENA. }\end{array}$ & $\begin{array}{l}\text { Activida- } \\
\text { des de } \\
\text { innovación }\end{array}$ \\
\hline
\end{tabular}

Fuente: Dirección de Formación Profesional del SENA.

Tabla 5. Ejecución de recursos Ley 344 de 1996 según programa (2002-2013)*

\begin{tabular}{|l|r|r|r|r|r|r|r|r|r|r|r|r|}
\hline \multicolumn{1}{|c|}{ Programa } & $\mathbf{2 0 0 2}$ & $\mathbf{2 0 0 3}$ & $\mathbf{2 0 0 4}$ & $\mathbf{2 0 0 5}$ & $\mathbf{2 0 0 6}$ & $\mathbf{2 0 0 7}$ & $\mathbf{2 0 0 8}$ & $\mathbf{2 0 0 9}$ & $\mathbf{2 0 1 0}$ & $\mathbf{2 0 1 1}$ & $\mathbf{2 0 1 2}$ & $\mathbf{2 0 1 3}$ \\
\hline $\begin{array}{l}\text { Modernización de la oferta de formación } \\
\text { profesional en centros de formación. } \\
\text { Incluye parques tecnológicos }\end{array}$ & 24.935 & 28.598 & 30.429 & 53.393 & 46.586 & 44.616 & 64.043 & 102.036 & 80.033 & 82.625 & 50.388 & 48.834 \\
\hline Innovación y Desarrollo tecnológico & 9.798 & 16.423 & 11.863 & 8.629 & 7.642 & 10.958 & 16.374 & 12.514 & 11.240 & 15.016 & 48.512 & 47.016 \\
\hline Alianza SENA - Colciencias & - & 15.000 & 21.828 & 36.481 & 40.123 & 46.025 & 54.545 & 31.495 & 100.367 & 80.155 & 86.224 & 86.224 \\
\hline Sena Digital & 59.015 & 902 & 25.900 & 18.938 & 19.830 & 25.613 & 35.159 & 53.902 & 82.887 & 57.896 & 99.392 & 88.169 \\
\hline Internacionalización & - & 1.500 & 893 & 860 & 1.636 & 1.763 & 8.189 & 1.000 & - & 3.167 & - & - \\
\hline Total & $\mathbf{9 3 . 7 4 8}$ & $\mathbf{6 2 . 4 2 3}$ & $\mathbf{9 0 . 9 1 3}$ & $\mathbf{1 1 8 . 3 0 1}$ & $\mathbf{1 1 5 . 8 1 7}$ & $\mathbf{1 2 8 . 9 7 5}$ & $\mathbf{1 7 8 . 3 1 0}$ & $\mathbf{2 0 0 . 9 4 7}$ & $\mathbf{2 7 4 . 5 2 7}$ & $\mathbf{2 3 8 . 8 5 9}$ & $\mathbf{2 8 4 . 5 1 6}$ & $\mathbf{2 7 0 . 2 4 3}$ \\
\hline
\end{tabular}

* Cifras en millones de pesos colombianos.

Fuente: Dirección de Planeación y Direccionamiento Corporativo, Dirección Administrativa y Financiera SENA, cálculos propios. 
Frente a lo anterior, se evidencia una asignación importante de recursos a las estrategias de competitividad y desarrollo tecnológico del SENA, lo cual genera resultados en diferentes campos de acción según los objetivos, los programas y las instancias de la entidad responsables de su implementación. No obstante, en lo anterior no se observa una política que contenga vasos comunicantes entre las diferentes iniciativas para la generación de sinergias entre programas, lo que resulta necesario para alinear las diferentes acciones hacia propósitos, objetivos y resultados generales en el ámbito de CTI.

En este contexto, con el ánimo de generar una mayor articulación interna y externa con los diferentes actores, especialmente con la política pública, en 2014 el SENA creó el Sistema de Investigación, Innovación y Desarrollo Tecnológico SENNOVA, a través del cual se ejecuta la política de contribución del SENA a la ciencia y tecnología del país de forma integral, fortaleciendo capacidades locales en productividad, competitividad y generación de conocimiento y pertinencia a través de la formación profesional impartida en la institución. Se observa que el concepto se acerca al proceso de creación, difusión y uso de las innovaciones (Pujol \& Arguimbau 2008), aproximándose al concepto de sistema que toma fuerza en la política pública del país y el ámbito internacional (Navarro et al. 2010; UNESCO, 2018). SENNOVA comprende tres programas y diez líneas programáticas según los principales objetivos de la entidad (tabla 6).

Tabla 6. Programas del SENNOVA (2014-2020)

\begin{tabular}{|c|c|c|c|}
\hline Programa & Línea & Objetivo & $\begin{array}{c}\text { Clasificación } \\
\text { ACTI }\end{array}$ \\
\hline \multirow{3}{*}{ Innovación } & $\begin{array}{l}\text { Cultura en Ciencia, } \\
\text { Tecnología e Innovación }\end{array}$ & $\begin{array}{l}\text { Fortalecer e incentivar actividades de investigación aplica- } \\
\text { da, desarrollo tecnológico e innovación en los centros de } \\
\text { formación por medio de publicaciones científicas, gestión } \\
\text { de eventos de divulgación y diversas acciones para promo- } \\
\text { ver la cultura I+D+i en las regiones. }\end{array}$ & $\begin{array}{l}\text { Actividades de } \\
\text { innovación }\end{array}$ \\
\hline & \multirow{2}{*}{$\begin{array}{l}\text { Fomento a la Innovación } \\
\text { y Desarrollo } \\
\text { Productivo en alianza } \\
\text { con las Empresas y } \\
\text { Centros de Formación }\end{array}$} & $\begin{array}{l}\text { En centros de formación SENA: generar alternativas de so- } \\
\text { lución de alta aplicabilidad a las necesidades y apuestas de } \\
\text { productividad y competitividad de las regiones mediante } \\
\text { financiación de proyectos de I+D+i en alianza con empresas } \\
\text { y entidades con presencia territorial. }\end{array}$ & $\begin{array}{l}\text { Actividades de } \\
\text { innovación }\end{array}$ \\
\hline & & $\begin{array}{l}\text { En el sector productivo: realizar acciones que contribuyan al } \\
\text { fortalecimiento de los procesos de investigación, desarrollo } \\
\text { tecnológico e innovación en el sector productivo colombia- } \\
\text { no, cuyos resultados incidan sobre los niveles de producti- } \\
\text { vidad y competitividad del mismo, así como en la formación } \\
\text { profesional integral y la formación para el trabajo. }\end{array}$ & $\begin{array}{l}\text { Actividades de } \\
\text { innovación }\end{array}$ \\
\hline \multirow[b]{2}{*}{ Investigación } & $\begin{array}{l}\text { Investigación Aplicada y } \\
\text { Semilleros de } \\
\text { Investigación en Centros } \\
\text { de Formación Grupos y } \\
\text { Semilleros. }\end{array}$ & $\begin{array}{l}\text { Generar capacidades de ciencia, tecnología e innovación en } \\
\text { los centros de formación SENA a través de grupos y semi- } \\
\text { lleros de investigación, como estrategias para fortalecer la } \\
\text { formación profesional y contribuir a la productividad, com- } \\
\text { petitividad y generación de conocimiento en las regiones. }\end{array}$ & $\begin{array}{l}\text { Investigación y } \\
\text { Desarrollo Expe- } \\
\text { rimental (I+D): }\end{array}$ \\
\hline & $\begin{array}{l}\text { Gestión del } \\
\text { Conocimiento }\end{array}$ & $\begin{array}{l}\text { Promover un enfoque colaborativo e integrado para la iden- } \\
\text { tificación, creación, captura, codificación, comunicación, } \\
\text { utilización, y almacenamiento de los activos intelectuales } \\
\text { del SENA de manera efectiva y para que } \\
\text { generen el mayor valor posible en los Centros de Formación } \\
\text { y en las empresas. }\end{array}$ & $\begin{array}{l}\text { Actividades de } \\
\text { innovación }\end{array}$ \\
\hline
\end{tabular}




\begin{tabular}{|c|c|c|c|}
\hline Programa & Línea & Objetivo & $\begin{array}{c}\text { Clasificación } \\
\text { ACTI }\end{array}$ \\
\hline \multirow{5}{*}{$\begin{array}{l}\text { Desarrollo } \\
\text { tecnológico }\end{array}$} & Tecnoparque & $\begin{array}{l}\text { Desarrollar proyectos que materialicen los conceptos de } \\
\text { proyectos de investigación aplicada, desarrollo tecnoló- } \\
\text { gico e innovación en prototipos funcionales y productos } \\
\text { que promuevan el emprendimiento de base tecnológica, la } \\
\text { productividad de las empresas y la transferencia de cono- } \\
\text { cimientos. }\end{array}$ & $\begin{array}{l}\text { Actividades de } \\
\text { innovación }\end{array}$ \\
\hline & Tecnoacademia & $\begin{array}{l}\text { Desarrollar habilidades, destrezas y competencias orienta- } \\
\text { das a la innovación, la ciencia y la tecnología en ambien- } \\
\text { tes dotados por tecnologías emergentes que fomenten en } \\
\text { niños y jóvenes de educación básica y media su vocación } \\
\text { hacía la CTI. }\end{array}$ & $\begin{array}{l}\text { Actividades de } \\
\text { innovación }\end{array}$ \\
\hline & $\begin{array}{l}\text { Fortalecimiento de } \\
\text { la Oferta de Servicios } \\
\text { Tecnológicos para las } \\
\text { Empresas }\end{array}$ & $\begin{array}{l}\text { Fortalecer la oferta de servicios tecnológicos para el sector } \\
\text { productivo y el SENA, orientados a la solución de fallas de } \\
\text { mercado, articulación, gobierno o programas de formación, } \\
\text { que sean pertinentes con la misionalidad de la entidad. }\end{array}$ & $\begin{array}{l}\text { Actividades de } \\
\text { innovación }\end{array}$ \\
\hline & $\begin{array}{l}\text { Actualización y } \\
\text { Modernización } \\
\text { Tecnológica de los } \\
\text { Centros de Formación }\end{array}$ & $\begin{array}{l}\text { Desarrollar proyectos orientados a la adopción de tecno- } \\
\text { logías y actualización de ambientes, apoyando el fortaleci- } \\
\text { miento de proyectos de I+D+i ejecutados por los grupos de } \\
\text { investigación y favoreciendo el mejoramiento tecnológico } \\
\text { de las herramientas y condiciones de los ambientes para } \\
\text { responder a los procesos de calidad en los programas de } \\
\text { formación de los Centros del SENA. }\end{array}$ & $\begin{array}{l}\text { Actividades de } \\
\text { innovación }\end{array}$ \\
\hline & $\begin{array}{l}\text { Extensionismo } \\
\text { Tecnológico }\end{array}$ & $\begin{array}{l}\text { Desarrollar y fortalecer las capacidades locales en transfe- } \\
\text { rencia tecnológica, a centros de formación, entidades para } \\
\text { capacitar extensionistas y el escalamiento de la metodolo- } \\
\text { gía en las empresas del sector productivo a través de exten- } \\
\text { sionistas tecnológicos. }\end{array}$ & $\begin{array}{l}\text { Actividades de } \\
\text { innovación }\end{array}$ \\
\hline
\end{tabular}

Fuente: Grupo SENNOVA.

Adicional a la articulación de los diferentes programas en torno a objetivos de política de CTI, en la estructura de SENNOVA se promovió la conformación de grupos y semilleros de investigación en los diferentes centros de formación del SENA, los cuales constituyen el brazo operativo para el desarrollo de los proyectos que se ejecutan internamente en la entidad, junto con el establecimiento de tecnoparques, tecnoacademias y laboratorios en los centros de formación del país. Además de lo anterior, se conformaron equipos de profesionales en cada uno de estos centros con instructores investigadores, gestores y líderes, quienes contribuyen a la implementación de las estrategias diseñadas. De igual manera, existen proyectos que se desarrollan con organizaciones del sector productivo y actores del ecosistema de CTI, los cuales mantienen una constante interacción con los centros de formación, grupos y semilleros de investigación en beneficio de los objetivos de la política institucional, particularmente en lo relacionado con la formación para el trabajo.

De acuerdo con Silva (2012), en los centros de formación del SENA se contaba con 28 grupos de investigación conformados y funcionando, de los cuales 6 tenían registro ante Colciencias y 2 manifestaron encontrarse en el proceso de obtención de dicho registro. De los grupos registrados en Colciencias, 1 contaba con categorización $\mathrm{D}$. Por regiones, estos se encontraban en Antioquia (2), Bolívar (1), Boyacá (3), 
Cauca (1), Cundinamarca (3), Bogotá (2), Huila (1), Quindío (2), San Andrés (1), Santander (8) y Valle del Cauca (4). A partir de la implementación de la estrategia SENNOVA se logró un cambio significativo, por lo que en la actualidad los 117 centros de formación del SENA cuentan con al menos un grupo de investigación, para un total de 119 grupos, ubicados en los 33 departamentos del país. En la más reciente convocatoria de reconocimiento y medición de grupos de investigación de Colciencias (2018), del total de grupos de investigación del SENA, se obtuvo la categorización de 1 grupo en la categoría A, 9 grupos en la categoría B y 75 en C, logrando además el reconocimiento de 2 grupos más (sin categorización), para un total de 89 grupos registrados.

En cuanto a la administración de recursos, la tabla 7 precisa el presupuesto ejecutado por SENNOVA entre 2014 y 2020 según el tipo de programa. Esta información permite observar una inversión total de COP 873.000 millones, de los cuales $7 \%$ corresponde a investigación y desarrollo experimental y $93 \%$ a actividades de innovación. Este monto resulta significativo si se compara con la inversión pública en ACTI reportada por universidades o centros de desarrollo tecnológico.

Tabla 7. Ejecución de recursos por parte del SENA SENNOVA según programa (2014-2020**

\begin{tabular}{|l|l|l|l|l|l|l|l|}
\hline Línea programática & 2014 & 2015 & 2016 & 2017 & 2018 & 2019 & 2020 \\
\hline Actualización y modernización tecnológica de los centros de formación & - & 20.000 & 31.749 & 29.094 & 23.785 & 35.172 & 35.014 \\
\hline Extensionismo tecnológico & - & 2.565 & - & 4.411 & 1.000 & 4.000 & 4.095 \\
\hline Cultura en CTI & 449 & 6.019 & 9.532 & 9.624 & 10.698 & 12.215 & 12.950 \\
\hline $\begin{array}{l}\text { Fomento de la innovación y desarrollo tecnológico en las empresas - } \\
\text { Centros SENA }\end{array}$ & - & 7.641 & 10.508 & 10.205 & 11.249 & 12.352 & 6.272 \\
\hline $\begin{array}{l}\text { Fomento de la innovación y desarrollo tecnológico en las empresas - } \\
\text { Sector productivo }\end{array}$ & 12.120 & 23.828 & 24.537 & 25.220 & 22.865 & 26.525 & 32.210 \\
\hline Fortalecimiento de la oferta de servicios tecnológicos para las empresas & 7.329 & 21.902 & 23.387 & 22.414 & 23.949 & 24.276 & 20.494 \\
\hline Gestión del conocimiento & - & - & - & 422 & 3.469 & 3.673 & 3.641 \\
\hline $\begin{array}{l}\text { Investigación aplicada y semilleros de investigación en centros de for- } \\
\text { mación }\end{array}$ & 4.000 & 8.120 & 9.414 & 9.313 & 9.659 & 11.431 & 11.422 \\
\hline Tecnoparque & 13.821 & 18.447 & 14.279 & 10.965 & 15.800 & 15.351 & 15.923 \\
\hline Tecnoacademia & - & - & 6.232 & 8.578 & 15.171 & 22.214 & 19.560 \\
\hline Total & $\mathbf{3 7 . 7 1 9}$ & $\mathbf{1 0 8 . 5 2 2}$ & $\mathbf{1 2 9 . 6 3 8}$ & $\mathbf{1 3 0 . 2 4 6}$ & $\mathbf{1 3 7 . 6 4 5}$ & 167.209 & 161.581 \\
\hline
\end{tabular}

* Cifras en millones de pesos colombianos.

Fuente: Dirección Administrativa y Financiera del SENA, cálculos propios.

En materia de resultados, a continuación se reseñan algunos aspectos de la inversión de los recursos en proyectos ejecutados por los grupos de investigación y los tecnoparques y tecnoacademias de los centros de formación. La tabla 8 precisa el número de proyectos realizados por centros de formación y sus grupos y semilleros de investigación, así como los ejecutados por tecnoparques, tecnoacademias y laboratorios del SENA. En concordancia con el paulatino proceso de implementación de SENNOVA, y a medida que se van generando sinergias entre los actores que participan en la estrategia, en general, se observa un crecimiento en el número de proyectos por programa y en el total. Frente a la clasificación ACTI, aproximadamente $30 \%$ de los proyectos se concentra en investigación y desarrollo experimental y el $70 \%$ restante en actividades de innovación. 
Tabla 8. Número de proyectos desarrollados en SENNOVA por línea programática (2017-2019)

\begin{tabular}{|c|c|c|c|}
\hline Programa & 2017 & 2018 & 2019 \\
\hline Actualización y modernización tecnológica de los centros de formación & 96 & 160 & 131 \\
\hline Cultura en ciencia, tecnología e innovación & 112 & 118 & 144 \\
\hline Fomento de la innovación y desarrollo tecnológico en las empresas - Centros SENA & 158 & 211 & 209 \\
\hline Fomento de la innovación y desarrollo tecnológico en las empresas - Convocatoria externa & 121 & 97 & 127 \\
\hline Fortalecimiento de la oferta de servicios tecnológicos para las empresas & 55 & 54 & 70 \\
\hline Investigación aplicada y semilleros de investigación en centros de formación grupos y semilleros & 204 & 228 & 242 \\
\hline Total & 625 & 771 & 796 \\
\hline
\end{tabular}

Fuente: Grupo SENNOVA.

Según el área de conocimiento de los proyectos, entre 2017 y 2019 el mayor porcentaje de las iniciativas ejecutadas se concentra en ingeniería, arquitectura, urbanismo y afines $(52,4 \%)$, seguido de agronomía, veterinaria y afines $(14,4 \%)$, ciencias de la educación $(7,8 \%)$, matemáticas y ciencias naturales (7,1\%), economía, administración, contaduría y afines $(5,9 \%)$ y bellas artes $(4,3 \%)$. Las ciencias de la salud y las ciencias sociales y humanas registran una participación de $4 \%$, cada una.

Tabla 9. Número de proyectos desarrollos en SENNOVA por área del conocimiento (2017-2019)

\begin{tabular}{|l|c|c|}
\hline \multicolumn{1}{|c|}{ Área de conocimiento } & $\begin{array}{c}\text { Número de } \\
\text { proyectos }\end{array}$ & $\%$ \\
\hline $\begin{array}{l}\text { Ingeniería, arquitectura, urbanismo y } \\
\text { afines }\end{array}$ & 1.149 & 52,4 \\
\hline Agronomía, veterinaria y afines & 316 & 14,4 \\
\hline Ciencias de la educación & 172 & 7,8 \\
\hline Matemáticas y ciencias naturales & 156 & 7,1 \\
\hline $\begin{array}{l}\text { Economía, administración, contaduría y } \\
\text { afines }\end{array}$ & 129 & 5,9 \\
\hline Bellas artes & 94 & 4,3 \\
\hline Ciencias de la salud & 88 & 4,0 \\
\hline Ciencias sociales y humanas & 88 & 4,0 \\
\hline Total general & 2.192 & 100 \\
\hline
\end{tabular}

Fuente: Grupo SENNOVA.

Al analizar los resultados por departamento, se observa una distribución relativamente equitativa entre 2017 y 2019, en el sentido en que todas las regiones del país desarrollan proyectos SENNOVA en las diferentes líneas programáticas. Adicionalmente, las cifras muestran que todos los grupos de investigación participan en estos proyectos y la mayoría de ellos desarrolla un número de proyectos cercano al promedio nacional (18).

Tabla 10. Número de proyectos desarrollos en SENNOVA por región y número de grupos de investigación (2017-2019)

\begin{tabular}{|l|c|c|c|}
\hline Departamento & $\begin{array}{c}\text { Grupos de } \\
\text { investigación }\end{array}$ & $\begin{array}{c}\text { Número de } \\
\text { proyectos }\end{array}$ & $\begin{array}{c}\text { Promedio } \\
\text { por grupo }\end{array}$ \\
\hline Amazonas & 1 & 16 & 16 \\
\hline Antioquia & 16 & 266 & 17 \\
\hline Arauca & 1 & 17 & 17 \\
\hline Atlántico & 4 & 116 & 29 \\
\hline Bolívar & 4 & 79 & 20 \\
\hline Boyacá & 4 & 71 & 18 \\
\hline Caldas & 5 & 114 & 23 \\
\hline Caquetá & 1 & 15 & 15 \\
\hline Casanare & 1 & 21 & 21 \\
\hline Cauca & 3 & 43 & 14 \\
\hline Cesar & 3 & 61 & 20 \\
\hline Choco & 1 & 15 & 15 \\
\hline Córdoba & 3 & 46 & 15 \\
\hline Cundinamarca & 6 & 124 & 21 \\
\hline Distrito Capital & 16 & 263 & 16 \\
\hline Guainía & 1 & 12 & 12 \\
\hline Guajira & 2 & 37 & 19 \\
\hline Guaviare & 1 & 15 & 15 \\
\hline Huila & 5 & 94 & 19 \\
\hline Magdalena & 2 & 31 & 16 \\
\hline Meta & 2 & 34 & 17 \\
\hline Nariño & 1 & 75 & 19 \\
\hline Norte de Santander & 2 & 46 & 23 \\
\hline Putumayo & & 16 & 16 \\
\hline Quindío & 52 & 17 \\
\hline
\end{tabular}




\begin{tabular}{|l|c|c|c|}
\hline \multicolumn{1}{|c|}{ Departamento } & $\begin{array}{c}\text { Grupos de } \\
\text { investigación }\end{array}$ & $\begin{array}{c}\text { Número de } \\
\text { proyectos }\end{array}$ & $\begin{array}{c}\text { Promedio } \\
\text { por grupo }\end{array}$ \\
\hline Risaralda & 3 & 60 & 20 \\
\hline San Andrés & 1 & 10 & 10 \\
\hline Santander & 8 & 174 & 22 \\
\hline Sucre & 1 & 23 & 23 \\
\hline Tolima & 3 & 62 & 21 \\
\hline Valle & 12 & 151 & 13 \\
\hline Vaupés & 1 & 15 & 15 \\
\hline Vichada & 1 & 18 & 18 \\
\hline Total general & 122 & 2.192 & 18 \\
\hline
\end{tabular}

El anterior resultado refleja un aspecto positivo de la distribución de los recursos, toda vez que para la aprobación de los proyectos existe un proceso de evaluación que verifica aspectos de la formulación. En este sentido, las regiones de menor desarrollo tienen las mismas oportunidades de recibir apoyo para sus iniciativas que las demás regiones del país.

Fuente: Grupo SENNOVA.

Con respecto al programa de innovación y desarrollo tecnológico que ejecutan las empresas, el SENA, a través de SENNOVA, ejecutó un rediseño a partir de las recomendaciones de la evaluación de impacto realizada por Econometría Consultores (2014), con lo cual en las convocatorias realizadas a partir de 2015 se logró incrementar el número de empresas beneficiadas, así como la participación de las micro y pequeñas empresas (tabla 11).

Tabla 11. Proyectos de innovación y desarrollo tecnológico ejecutados en empresas

\begin{tabular}{|c|c|c|c|c|c|c|}
\hline \multirow[b]{2}{*}{ Departamento } & \multicolumn{2}{|c|}{$2010-2014$} & \multicolumn{2}{|c|}{$2016-2018$} & \multicolumn{2}{|c|}{$2019-2020$} \\
\hline & Proyectos & Cofinanciación* & Proyectos & Cofinanciación* & $\begin{array}{c}\text { Proyec- } \\
\text { tos }\end{array}$ & Cofinanciación* \\
\hline Amazonas & - & - & 2 & 274.355 .800 & 1 & 200.000 .000 \\
\hline Antioquia & 80 & 12.721 .621 .310 & 28 & 5.242.662.179 & 24 & 4.278 .180 .727 \\
\hline Arauca & - & - & 2 & 387.129 .327 & 2 & 398.680 .000 \\
\hline Atlántico & 10 & 1.793.040.180 & 24 & 4.710.097.769 & 15 & 2.939.776.034 \\
\hline Bogotá D.C. & 64 & 16.100 .740 .813 & 28 & 5.520 .010 .057 & 39 & 7.303 .525 .080 \\
\hline Bolívar & 13 & 2.757.150.989 & 10 & 1.718 .448 .258 & 6 & 1.126 .510 .000 \\
\hline Boyacá & 2 & 320.556 .838 & 10 & 1.727 .341 .875 & 6 & 1.087.779.356 \\
\hline Caldas & 9 & 1.113 .394 .748 & 14 & 1.995 .085 .304 & 6 & 1.198 .723 .608 \\
\hline Caquetá & - & - & 6 & 1.091 .115 .640 & 1 & 161.500 .382 \\
\hline Casanare & - & - & 6 & 508.062 .553 & 2 & 353.655 .272 \\
\hline Cauca & 1 & 167.672 .100 & 7 & 984.941 .511 & 6 & 1.115 .092 .722 \\
\hline Cesar & 2 & 76.300 .000 & 5 & 926.376 .981 & 4 & 781.009 .135 \\
\hline Chocó & - & - & 6 & 688.094 .000 & 2 & 392.741 .779 \\
\hline Córdoba & - & - & 4 & 698.847 .452 & 5 & 990.012 .043 \\
\hline Cundinamarca & 11 & 1.464.195.274 & 6 & 1.098 .605 .894 & 6 & 1.137 .186 .000 \\
\hline Guainía & - & - & 3 & 246.060 .000 & - & - \\
\hline Guaviare & - & - & 1 & 103.040 .620 & - & - \\
\hline Huila & 5 & 1.378.251.173 & 11 & 2.239.510.981 & 7 & 1.346.753.204 \\
\hline La Guajira & 3 & 423.458 .800 & 6 & 810.304 .708 & 1 & 200.000 .000 \\
\hline Magdalena & 1 & 60.881 .000 & 5 & 791.367 .650 & 5 & 975.666 .811 \\
\hline Meta & 3 & 367.559 .687 & 5 & 632.616 .046 & 6 & 1.158 .377 .685 \\
\hline Nariño & 6 & 1.022 .336 .028 & 9 & 1.339.267.030 & 6 & 1.069.358.088 \\
\hline Norte de Santander & - & - & 6 & 1.125 .035 .532 & 4 & 713.573 .064 \\
\hline Putumayo & - & - & 5 & 979.738 .916 & 1 & 199.260 .000 \\
\hline
\end{tabular}




\begin{tabular}{|c|c|c|c|c|c|c|}
\hline \multirow[b]{2}{*}{ Departamento } & \multicolumn{2}{|c|}{ 2010-2014 } & \multicolumn{2}{|c|}{ 2016-2018 } & \multicolumn{2}{|c|}{ 2019-2020 } \\
\hline & Proyectos & Cofinanciación* & Proyectos & Cofinanciación* & $\begin{array}{l}\text { Proyec- } \\
\text { tos }\end{array}$ & Cofinanciación* \\
\hline Quindío & 1 & 94.373 .507 & 9 & 1.279 .813 .725 & 6 & 1.045 .634 .356 \\
\hline Risaralda & 10 & 2.584.547.107 & 31 & 5.050 .104 .198 & 18 & 3.380 .672 .533 \\
\hline $\begin{array}{l}\text { San Andrés y Providen- } \\
\text { cia }\end{array}$ & - & - & 4 & 691.793 .068 & - & - \\
\hline Santander & 8 & 3.881 .652 .639 & 24 & 4.762 .903 .822 & 14 & 2.728 .331 .300 \\
\hline Sucre & - & - & 5 & 836.865 .968 & 3 & 595.100 .000 \\
\hline Tolima & 3 & 274.019 .866 & 11 & 1.858 .794 .480 & 6 & 1.190 .364 .852 \\
\hline Valle del Cauca & 6 & 1.266 .101 .473 & 29 & 4.744 .041 .428 & 21 & 3.986 .101 .121 \\
\hline Vaupés & - & - & 2 & 131.872 .580 & - & - \\
\hline Vichada & - & - & 2 & 314.254 .900 & 1 & 195.030 .514 \\
\hline Total & 238 & 47.867 .853 .532 & 326 & 55.508 .560 .252 & 224 & 42.248 .595 .666 \\
\hline
\end{tabular}

* Cifras en pesos colombianos.

Fuente: Grupo SENNOVA.

Entre 2016 y 2018, SENNOVA, a través de este programa, aprobó proyectos en los 33 departamentos del país, cuando entre 2010 y 2014 llegó a solo 20 departamentos, con una concentración significativa en Bogotá y Antioquia. Para los procesos realizados en 2019-2020 se lograron resultados similares en cobertura y tamaños de empresas. Estos resultados se obtuvieron a través del diseño de acciones regionales y la vinculación de expertos al ecosistema de CTI, con el fin de apoyar la generación de capacidades en formulación y gestión de proyectos (2016-2018), iniciativa en la que también participaron Minciencias y el Ministerio de Industria, Comercio y Turismo (2019-2020). Adicionalmente, con ocasión de la ejecución de proyectos conjuntos con empresas, los centros de formación participaron en procesos de transferencia de tecnología que incluyeron la participación de aprendices e instructores, la realización de pasantías, la donación de prototipos y la adquisición de material para diseño o actualización de programas, entre otros.

Con base en los resultados señalados, se evidencia el relacionamiento entre los grupos y semilleros de investigación, especialmente, así como entre los demás programas institucionales, actores e instituciones del ecosistema de CTI, formando la aparición de laboratorios ciudadanos similares a algunas experiencias de ciencia publica y participativa, tendencia que comienza a marcarse y que tendrá influencia en el diseño futuro de política de CTI (Meissner et al., 2021).
De manera particular, se resalta el beneficio para la formación profesional, que mediante los diferentes programas permite la generación y actualización de conocimientos para aprendices, instructores y programas. Sumado a ello, a través de la interacción con otros instrumentos, entidades y programas de gobierno, se contribuye a generar sinergias y fomentar la eficiencia en el gasto público en CTI en Colombia.

\section{CONCLUSIONES Y RECOMENDACIONES}

A nivel conceptual, aunque con diferentes matices y enfoques, se reconoce la importancia de combinar factores productivos, de capital y de trabajo, así como a la ciencia y la tecnología, en la transformación del aparato productivo, lo cual resulta fundamental dentro de las políticas de desarrollo y además propicia la vinculación de agentes con diferentes intereses, pero que trabajan en la ejecución de acciones interrelacionadas.

A nivel de política pública en Latinoamérica, en las últimas décadas se observa una evolución que va de programas focalizados en formación científica (un poco desconectada con las necesidades reales) al diseño de sistemas de CTI con énfasis en la generación de incentivos adecuados, los cuales facilitan la integración y convergencia de intereses entre los diferentes agentes involucrados. 
Frente a los resultados de la política pública, se observa que la inversión es considerablemente inferior a la de países europeos o aquellos que son miembros de la Organización para la Cooperación y el Desarrollo Económico (OCDE). Sin embargo, pese a las deficiencias y características de la inversión en innovación, algunos estudios señalan un resultado positivo sobre la productividad, aunque con efectos tardíos, en algunos casos. Dentro de las posibles explicaciones a esta situación se puede atribuir la capacidad del capital humano para adaptarse a los nuevos desarrollos, la preferencia del sector productivo por inversiones con rendimientos rápidos, los costos asociados al aprendizaje y la inclinación de las empresas a realizar procesos de innovación en períodos de desaceleración económica, entre otros.

En cuanto a la formación profesional, la evidencia internacional señala su integración con elementos de desarrollo tecnológico, asistencia técnica, consultoría, diseño, investigación aplicada, pruebas y ensayos de laboratorio, entre otros. En ese sentido, y teniendo en cuenta los planteamientos de política pública en cuanto a sistemas de ciencia, tecnología e innovación, SENNOVA constituye una estrategia que se encuentra alineada con objetivos institucionales y de política pública, por lo que es razonable dar continuidad y mayor profundidad a esta iniciativa. En efecto, a partir de la conformación de este sistema se observa que - aunque la mayoría de acciones se concentra en actividades de innovación- la importancia del desarrollo de proyectos de investigación y de desarrollo experimental, a la par con el fortalecimiento de los grupos de investigación, es notable, toda vez que estos no tenían mayor participación en el pasado. Por otra parte, se observa que las acciones en innovación, investigación y desarroIlo experimental del SENA guardan coherencia con la tendencia a conformar sistemas que fortalezcan su vínculo con la formación del recurso humano y el sector productivo.

Con base en los resultados y su articulación con políticas locales e internacionales, la estrategia SENNOVA constituye un referente en la formulación e implementación de política en CTI. En particular, SENNOVA ha fomentado la apropiación tecnológica, social y colectiva al transferir el conocimiento generado en los proyectos de los diferentes programas a los procesos de formación profesional, tanto para aprendices como instructores y demás actores del sector productivo en las diferentes regiones, contribuyendo así con la disminución de brechas tecnológicas en el país.

Por lo anterior, se recomienda llevar a cabo una evaluación de impacto que permita analizar cualitativa y cuantitativamente los resultados de esta estrategia institucional, con el objetivo de diseñar políticas de largo plazo que incidan sobre el desempeño de los diferentes actores públicos y privados y como un aporte a nuevas iniciativas y propuestas de política pública en CTI.

\section{REFERENCIAS}

Amankwah-Amoah, J. (2016). The evolution of science, technology and innovation policies: A review of the Ghanaian experience. Technological Forecasting \& Social Change, 110, 134-142. https://doi.org/10.1016/j.techfore.2015.11.022

Banco Interamericano de Desarrollo [BID] (2020). Respuestas al COVID-19 desde la ciencia, la innovación y el desarrollo productivo. BID.

Centro de Investigaciones para el Desarrollo [CID]. (2010). Evaluación de impactos de proyectos de investigación y desarrollo e innovación financiados por Colciencias en el periodo 19992005. CID.

Centro Interamericano para el Desarrollo del Conocimiento de la Formación Profesional [Cinterfor]. (2017). El fututo de la formación profesional en América Latina y el Caribe. OIT.

Cruz, M., Frias, J., laconove, L., Cirela, X., \& Medvedev, D. (2018). Análisis de eficiencia del gasto público en ciencia, tecnología e innovación en Colombia (documento de trabajo). Banco Mundial.

Congreso de Colombia. (27 de febrero de 1990). Ley 29 de 1990. Por la cual se dictan disposiciones para el fomento de la investigación científica y el desarrollo tecnológico y se otorgan facultades extraordinarias. Diario Oficial 39205. 
Congreso de Colombia. (23 de enero de 2009). Ley 1286 de 2009. Por la cual se modifica la Ley 29 de 1990, se transforma a Colciencias en Departamento Administrativo, se fortalece el Sistema Nacional de Ciencia, Tecnología e Innovación en Colombia y se dictan otras disposiciones. Diario Oficial 47241.

Congreso de Colombia. (24 de enero de 2019). Ley 1951 de 2019. Por la cual crea el ministerio de ciencia, tecnología e innovación, se fortalece el sistema nacional de ciencia, tecnología e innovación y se dictan otras disposiciones. Diario Oficial 50846.

Consejo Nacional de Política Económica y Social [Conpes]. (2009). Política Nacional de Ciencia, Tecnología e innovación. Conpes.

Consejo Nacional de Política Económica y Social [Conpes]. (2016). Política Nacional de Desarrollo Productivo. Conpes.

Consejo Nacional de Política Económica y Social [Conpes]. (2020). Política Nacional de Ciencia, Tecnología e Innovación 2021-2030. Conpes.

Departamento Nacional de Planeación [DNP]. (2019a). Bases del Plan Nacional de Desarrollo 2018-2022. Pacto por Colombia, pacto por la equidad. DNP.

Departamento Nacional de Planeación [DNP]. (2019b). Índice departamental de innovación para Colombia. DNP. https://colaboracion.dnp.gov.co/ CDT/Prensa/PresentacionIDICValledelCauca.pdf

Departamento Nacional de Planeación [DNP]. (2020). Lineamientos para la optimización y articulación de la oferta de instrumentos de política pública para la competitividad y la innovación (circular externa 02-4). DNP.

Departamento Administrativo Nacional de Estadística [DANE]. (2019), Boletín técnico encuesta de desarrollo e innovación tecnológica sector industria manufacturera 2017-2018. DANE.
Econometría Consultores. (2014). Evaluación Programa de Innovación de Innovación y Desarrollo Tecnológico (documento de trabajo). Econometría Consultores.

Fernández-Sastre, J., \& Montalvo-Quizhpi, F. (2019). The effect of developing countries' innovation policies on firms' decisions to invest in R\&D. Technological Forecasting \& Social Change, 143(2), 214-223. https://doi.org/10.1016/j. techfore.2019.02.006

Fundación Andina para el Desarrollo Tecnológico y Social [Tecnos] (2010). Informe ejecutivo, Evaluación de Impactos de Proyectos de Investigación y Desarrollo e Innovación financiados por Colciencias en el período 19992005 en la Región Occidente. Tecnos.

Hernández, I. (2004). Los modelos de difusión evolucionista. Una aproximación institucional. Cuadernos de Economía, 23(40), 79-110.

Ibáñez, J. (2018). La ciencia en Latinoamérica: tendencias y patrones. Revista de la Facultad de Ciencias, 7(1), 23-39. https://doi.org/10.15446/ rev.fac.cienc.v7n1.69409

inNpulsa Colombia. (2021). Fortalecemos a los emprendedores y empresarios de Colombia. https://www.innpulsacolombia.com/

Lugo, C. (2017). Los técnicos de la innovación. Universidad de Salamanca.

Maffioli, A., \& Meléndez, M. (2011). El uso de indicadores para la evaluación de programas de ciencia, tecnología e innovación: el caso de Colciencias (documento de trabajo). BID.

Meissner, D., Sarpong, D., Ofosu, G., \& Botchie, D. (2021). The rise of do-it-yourself (DiY) laboratories: Implications for science, technology, and innovation (STI) policy. Technological Forecasting \& Social Change, 165, 120589. https://doi.org/10.1016/j.techfore.2021.120589 
Ministerio de Ciencia Tecnología e Innovación [Minciencias]. (2020a). Beneficios tributarios apalancan $50 \%$ más de inversiones en CTel en el 2020. https://www.minciencias.gov. co/sala_de_prensa/beneficios-tributariosapalancan-50-mas-inversiones-en-ctei-enel-2020\#: :text=(\%40mincienciasCo)\%20 El\%20Consejo\%20Nacional,a\%20las\%20 empresas\%20en\%20alianza

Ministerio de Ciencia Tecnología e Innovación [Minciencias]. (2020b). Convocatoria Doctorados, Gobierno de Colombia. https:// minciencias.gov.co/convocatorias/formaciony-vinculacion-capital-humano-alto-nivel/ convocatoria-doctorados-en-el

Ministerio de Tecnologías de la Información y las Comunicaciones [MinTIC]. (2021). Apps.co. https://apps.co/765/w3-channel.html

Montoya, O. (2004). Schumpeter, innovación y determinismo tecnológico. Scientia et Technica, 2(25), 209-213.

Navarro, J., Llisterri, J., \& Zúñiga, P. (2010). La importancia de las ideas: innovación y productividad en América Latina. En C. Pagés (ed.), La era de la productividad. Cómo transformar las economías desde sus cimientos (pp. 265-303). BID.

Observatorio Colombiano de Ciencia y Tecnología [OCyT]. (2008). Cálculo de la inversión en actividades de ciencia, tecnología e innovación. Guía para el formulario de inversión a ACTI. OCYT

Observatorio Colombiano de Ciencia y Tecnología [OCYT]. (2019). Indicadores de ciencia y tecnología 2018. OCyT

Observatorio Colombiano de Ciencia y Tecnología [OCyT]. (2020). Indicadores de ciencia y tecnología 2019. OCyT.
Organización de las Naciones Unidas para la Educación, la Ciencia y la Cultura [UNESCO]. (2018). Informe de la UNESCO sobre la Ciencia, hacia 2030: informe regional de América Latina y el Caribe. UNESCO.

Pagés, C. (ed.). (2010). La era de la productividad. Cómo transformar las economías desde sus cimientos. BID.

Pardini, R., Bertuol-Garcia, D., Demasi-Araújo, B., Mesquita, J. P., Moraes-Murer, B., CarmoPonzio, M., Silvério-Ribero, F., Laganaro-Rossi, M., \& Prado, P. I., (2021). COVID-19 pandemic as a learning path for grounding conservation policies in science. Perspectives in Ecology and Conservation, 19(2), 109-114. https://doi. org/10.1016/j.pecon.2021.02.009

Peijie, W., \& Fan L. (2021). Science, technology and innovation policy in Russia and China - Mapping and comparisons in objectives, instruments and implementation. Technological Forecasting \& Social Change, 162, 120386. https://doi. org/10.1016/j.techfore.2020.120386

Presidencia de la República de Colombia. (8 de febrero de 1991). Decreto 393 de 1991. Por el cual se dictan normas sobre asociación para actividades científicas y tecnológicas, proyectos de investigación y creación de tecnologías. Diario Oficial 39672.

Presidencia de la República de Colombia. (26 de febrero de 1991). Decreto 585 de 1991. Por el cual se crea el consejo nacional de ciencia y tecnología, se reorganiza el Instituto Colombiano para el Desarrollo de la Ciencia y la Tecnología, Colciencias, y se dictan otras disposiciones. Diario Oficial 39702.

Presidencia de la República de Colombia. (18 de diciembre de 2019). Directiva Presidencial No. 12. Optimización y articulación de la oferta de instrumentos de política pública para la competitividad y la innovación. Diario Oficial 51701. 
Pujol, E., \& Arguimbau, L. (2008). I+D+l: Una perspectiva documental. Anales de Documentación, 11, 43-56.

Santa-María, M., Acosta, P., Estacio, A., Millán, N., Olivera, M., \& Parra, M. (2009). Evaluación de impacto de tres programas y línea de base de un programa del Servicio Nacional de AprendizajeSENA, Informe Final. Fedesarrollo.

SENNOVA. (2021). Sistema de gestión de información de proyectos. http://sennova.senaedu.edu.co/

Silva, M. (2012). Encuesta sobre grupos de investigación y políticas editoriales a los Centros de Formación Profesional (documento de trabajo, Dirección del Sistema Nacional de Formación para el Trabajo). SENA.
Sistema Nacional de Competitividad e Innovación [SNCI]. (2021). Articular para la competitividad, Gobierno de Colombia. http:// www.colombiacompetitiva.gov.co/prensa/ articular-para-la-competitividad

Universidad del Norte [UN], \& Universidad Industrial de Santander [UIS]. (2010). Evaluación de Impactos de Proyectos de Investigación y Desarrollo e Innovación financiados por Colciencias en el período 1999-2005 en la Región Nororiental. Informe Final. UN \& UIS.

Zerda, A. (2003). Ciencia y tecnología en el Plan de Desarrollo. Cuadernos de Economía, 22(39), 124-132. 